\title{
CONE AVOIDING CLOSED SETS
}

\author{
LU LIU
}

\begin{abstract}
We prove that for an arbitrary subtree $T$ of $2^{<\omega}$ with each element extendable to a path, a given countable class $\mathcal{M}$ closed under disjoint union, and any set $A$, if none of the members of $\mathcal{M}$ strongly $k$-enumerate $T$ for any $k$, then there exists an infinite set contained in either $A$ or $\bar{A}$ such that for every $C \in \mathcal{M}, C \oplus G$ also does not strongly $k$-enumerate $T$. We give applications of this result, which include: (1) $\mathrm{RT}_{2}^{2}$ doesn't imply WWKL $\mathrm{L}_{0}$; (2) [Ambos-Spies et al., 2004] DNR is strictly weaker than $W_{W K L}$; (3) [Kjos-Hanssen, 2009] for any Martin-Löf random set $A$, either $A$ or $\bar{A}$ contains an infinite subset that does not compute any Martin-Löf random set; etc. We also discuss further generalizations of this result.
\end{abstract}

\section{INTRODUCTION}

The interrelationship between topological properties and computability theoretic properties (usually computational power) of a class is widely studied in various branches of recursion theory. Here topological property is a fairly feeble term; for example such properties could involve

- all infinite homogeneous sets of a coloring of $[N]^{n}$;

- all infinite subsets of $A$ or $\bar{A}$;

- all paths through a tree $T$. For this case, different combinatorial or topological conditions on the tree yield different topological conditions on the corresponding class. For example: $T$ is finitely branching; homogeneous; $[T]$ is of positive measure, positive Hausdorff measure; etc.;

- all representations of a continuous function: $[0,1] \rightarrow[0,1]$.

This paper focuses on two kinds of classes, Part $_{k}$ and $[Q]$, where

$$
\begin{aligned}
& \operatorname{Part}_{k}\left(A_{1}, A_{2}, \ldots, A_{k-1}\right) \\
& \quad=\left\{X \in 2^{\omega}:\left(\exists i \leq k-1, X \subseteq A_{i} \vee X \subseteq \omega-\bigcup_{j=1}^{k-1} A_{j}\right) \wedge|X|=\infty\right\}
\end{aligned}
$$

and $[Q]$ is a closed set of $2^{\omega}$. On the positive side, Hirschfeldt et al. 10, proved that there exists $A \in \Delta_{2}^{0}$ such that $D N R \leq_{u} \operatorname{Part}_{2}(A)$, where $D N R=\left\{f \in \omega^{\omega}\right.$ : $\left.\varphi_{n}(n) \uparrow \vee \varphi_{n}(n) \neq f(n)\right\}$, and $\leq_{u}$ denotes the Muchnik reducibility (i.e. $\mathcal{C}_{2} \leq_{u} \mathcal{C}_{1}$ means $\forall X \in \mathcal{C}_{1} \exists Y \in \mathcal{C}_{2}$ such that $\left.Y \leq_{T} X\right)$, while on the negative side Dzhafarov and Jockusch [6] showed that for any $0<_{T} C$ and any $A \in 2^{\omega},\{C\} \not_{u} \operatorname{Part}_{2}(A)$. Kjos-Hanssen [13] showed that every real of positive effective Hausdorff dimension

Received by the editors November 20, 2010 and, in revised form, December 12, 2012.

2010 Mathematics Subject Classification. Primary 03B30; Secondary 03F35, 03C62, 68Q30, 03D32, 03D80, 28A78.

Key words and phrases. Ramsey's theorem, weak weak König's lemma, Martin-Löf randomness, randomness extraction. 
computes an infinite subset of a Martin-Löf random set, and Greenberg and Miller [9] showed the same for every $f$-DNR (an $f$-DNR is a $D N R g$ such that $(\forall n) g(n) \leq$ $f(n)$ ), where $f$ is a sufficiently "slow growing" computable function. Cholak et al. [4] showed that for any $A \in \Delta_{2}^{0}$ there exists an infinite low 2 set $G$ contained in either $A$ or $\bar{A}$.

We say we can cone avoid $\mathcal{C}_{2}$ within $\mathcal{C}_{1}$ iff $\mathcal{C}_{2} \not_{u} \mathcal{C}_{1}$. In this paper we generalize a result of the author [11, which proves the cone avoidance result for arbitrary Part $_{k}$ and $[Q]=2-D N R$ (where $2-D N R=\left\{X \in 2^{\omega}: \forall n \varphi_{n}(n) \downarrow \rightarrow \varphi_{n}(n) \neq X(n)\right\}$ ).

The following is the main theorem in this paper. For a closed set $[Q]$ (also denoted as $\mathcal{Q}$ ) of $2^{\omega}$ let $Q=\left\{\rho \in 2^{<\omega}:[\rho] \preceq \cap[Q] \neq \emptyset\right\}$ (where $[\rho] \preceq=\left\{X \in 2^{\omega}: X \supset \rho\right\}$ ).

Definition 1.1 (Beigel et al. 2]). Fix the canonical representation of finite sets, with each finite set denoted by $D_{n}$, where $n$ is the (canonical) index of this finite set. Let $k$ be a positive integer. Let $\left\{X_{i}\right\}_{i \in \omega}$ be an array of non-empty sets.

A strong $k$-enumeration of $\left\{X_{i}\right\}_{i \in \omega}$ is a function $h \in \omega^{\omega}$ such that $\left|D_{h(n)}\right| \leq k$ and $X_{n} \cap D_{h(n)} \neq \emptyset$. A strong constant-bound-enumeration of $\left\{X_{i}\right\}_{i \in \omega}$ is a strong $k$-enumeration for some $k$.

Let $Q$ be a set of strings, say a tree. In the following text we also regard $Q$ as an array of sets, i.e. $Q=\bigcup_{n \in \omega} Q_{n}$, where $Q_{n}$ is the set of $n$-length strings of $Q$. A strong $k$-enumeration of $Q$ means a strong $k$-enumeration of $\left\{Q_{i}\right\}_{i \in \omega}$.

Note that if $Q$ is a tree and $W \subseteq \omega$ is infinite, then a strong $k$-enumeration of $\left\{Q_{r_{n}}\right\}_{r_{n} \in W}$ can be effectively translated to a strong $k$-enumeration of $\left\{Q_{i}\right\}_{i \in \omega}$.

Theorem 1.2. Suppose $\mathcal{M}=\left\{C^{(1)}, C^{(2)}, \ldots\right\}$ is a countable class of sets and $\mathcal{Q}=[Q]$ is a closed set (of $2^{\omega}$ ) such that

- $\forall C^{(i)}, C^{(j)} \in \mathcal{M}, C^{(i)} \oplus C^{(j)} \in \mathcal{M}$ and

- $Q$ doesn't admit a strong constant-bound-enumeration computable in $\mathcal{M}$ (i.e. computable in some member of $\mathcal{M}$ ).

Then for any set $A$, there exists an infinite set $G$ such that

- $G \subseteq A \vee G \subseteq \bar{A}$ and

- $\forall C \in \mathcal{M}, G \oplus C$ also doesn't compute any strong constant-bound-enumeration of $Q$. In particular, $G \oplus C$ does not compute any member of $[Q]$, since otherwise clearly $G \oplus C$ computes a strong 1-enumeration of $Q$.

To gain some interest, we first introduce some applications in reverse mathematics and algorithm randomness theory, which will be derived from Theorem 1.2 in Section 5

Reverse mathematics studies the proof theoretic strength of various second order arithmetic statements. Several statements are so important and fundamental that they serve as level lines. Many mathematical theorems are found to be equivalent to these statements and are unchanged under small perturbations of themselves. The relationships between these statements and "other" statements draw much attention. $\mathrm{WKL}_{0}$ is one of these statements. $\mathrm{WKL}_{0}$ states that every infinite binary tree admits an infinite path. It is well known that as a second order arithmetic statement, $\mathrm{WKL}_{0}$ is equivalent to the statement that for any set $C$ there exists $B \gg C$, where $B \gg C$ means $B$ is of PA-degree relative to $C$. A good survey of reverse mathematics is [23] or [7, 8]. One of the second order arithmetic statements close to $\mathrm{WKL}_{0}$ is $\mathrm{RT}_{2}^{2}$. 
Definition 1.3. Let $[X]^{k}$ denote $\{F \subseteq X:|F|=k\}$. A $k$-coloring $f$ is a function $[X]^{n} \rightarrow\{1,2, \ldots, k\}$. A set $H \subseteq[X]^{k}$ is homogeneous for $f$ iff $f$ is constant on $[H]^{k}$. A stable coloring $f$ is a 2-coloring of $[\mathbb{N}]^{2}$ such that $(\forall n \in \mathbb{N})(\exists N)(\forall m>N)$ $f(\{m, n\})=f(\{N, n\})$. For a stable coloring $f, f_{1}=\{n \in \mathbb{N}:(\exists N)(\forall m>$ $N), f(m, n)=1\}, f_{2}=\mathbb{N}-f_{1}$.

Ramsey's theorem (Ramsey [20]). For any $n$ and $k$, every $k$-coloring of $[\mathbb{N}]^{n}$ admits an infinite homogeneous set.

Let $\mathrm{RT}_{k}^{n}$ denote Ramsey's theorem for $k$-colorings of $[\mathbb{N}]^{n}$ and $\mathrm{SRT}_{k}^{2}$ denote Ramsey's theorem restricted to a stable coloring of pairs. It is clear that $\mathrm{RT}_{k}^{2}$ implies $\mathrm{SRT}_{k}^{2}$.

Jockusch [12] showed that for $n>2, \mathrm{RT}_{2}^{n}$ is equivalent to $\mathrm{ACA}_{0}$, while Seetapun and Slaman $\left[22\right.$ showed that $\mathrm{RT}_{2}^{2}$ does not imply $\mathrm{ACA}_{0}$. As to $\mathrm{WKL}_{0}$, Jockusch [12 proved that $\mathrm{WKL}_{0}$ does not imply $\mathrm{RT}_{2}^{2}$. Whether $\mathrm{RT}_{2}^{2}$ implies $\mathrm{WKL}_{0}$ remained open. A more detailed survey of Ramsey's theorem in view of reverse mathematics can be found in Cholak, Jockusch and Slaman 4. Say a set $S$ cone avoids a class $\mathcal{M}$ iff $(\forall C \in \mathcal{M})\left[C \not \mathbb{Z}_{T} S\right]$.

This problem has been a major focus in reverse mathematics in the past twenty years. The first important progress was made by Seetapun and Slaman 22, where they showed that

Theorem 1.4 (Seetapun and Slaman [22]). For any countable class of sets $\left\{C_{j}\right\}$, $j \in \omega$, such that each $C_{i}$ is non-computable, any computable 2-coloring of pairs admits an infinite cone avoiding (for $\left\{C_{j}\right\}$ ) homogeneous set.

Parallel to this result, using Mathias forcing in a different manner, Dzhafarov and Jockusch [6. Lemma 3.2] proved that

Theorem 1.5 (Dzhafarov and Jockusch [6]). For any set $A$ and any countable class $\mathcal{M}$ such that each member of $\mathcal{M}$ is non-computable, there exists an infinite set $G$ contained in either $A$ or its complement such that $G$ is cone avoiding for $\mathcal{M}$.

The main idea is to restrict the computational complexity (computability power) of the homogeneous set as much as possible, with complexity measured by various measurements. Along this line, with simplicity measured by extent of lowness, Cholak, Jockusch and Slaman [4, Theorem 3.1] showed, by a fairly ingenious argument,

Theorem 1.6 (Cholak, Jockusch, and Slaman [4]). For any computable coloring of the unordered pairs of natural numbers with finitely many colors, there is an infinite low $_{2}$ homogeneous set $X$.

The author proved [11] that $\mathrm{RT}_{2}^{2}$ does not imply $\mathrm{WKL}_{0}$. Meanwhile, it had also been wondered whether $R T_{2}^{2}$ implies $W W K L_{0}$. Here $W W K L_{0}$ is a weaker version of $\mathrm{WKL}_{0}$ as follows.

Definition 1.7. WWKL $L_{0}: \forall T \subseteq 2^{<\omega}$, if $T$ is a tree s.t. $(\exists a>0 \exists b \forall n) \frac{\left|\left\{\rho \in T_{n}\right\}\right|}{2^{n}}>$ $\frac{a}{b}$, then there exists an infinite path $X \in[T]$.

Intuitively, WWKL $L_{0}$ states: for every infinite tree $T \subseteq 2^{<\omega}$, if $\mu([T])>0$, then there is a path through $T$. It is also considered as a statement in the language of 
second order arithmetic. For background on reverse mathematics see [23] and [4], which focus on the proof theoretic strength of Ramsey's theorem for pairs.

Our major application is that,

Corollary 1.8. Over $\mathrm{RCA}_{0}, \mathrm{RT}_{2}^{2}$ does not imply $\mathrm{WWKL}_{0}$.

Here $\mathrm{RCA}_{0}$ is the axiom which says that for any member $X$ of a model of arithmetic, all sets computable in $X$ should also be included in the model. This axiom corresponds to the wide intuition in mathematics that things that are derivable, constructible (in some sense) from a given object that is known to exist, should also be considered to exist.

Let $D N R=\left\{f \in \omega^{\omega}: \forall e, f(e) \neq \Phi_{e}(e) \vee \Phi_{e}(e) \uparrow\right\}, D N R^{X}=\left\{f \in \omega^{\omega}: \forall e, f(e) \neq\right.$ $\left.\Phi_{e}^{X}(e) \vee \Phi_{e}^{X}(e) \uparrow\right\}$, and $g-D N R=\left\{f \in \omega^{\omega}: f \in D N R \wedge(\forall n) f(n) \leq g(n)\right\}$. DNR as a second order arithmetic statement says: $\forall X \exists f \in \mathrm{DNR}^{X}$. Hirschfeldt et al. [10, Theorem 2.3] showed that $\mathrm{SRT}_{2}^{2}$ implies DNR; they constructed a $\Delta_{2}^{0}$ set $A$ such that any infinite subset of $A$ or $\bar{A}$ computes a $D N R$ function. It is also well known that $W_{W K L_{0}}$ implies DNR. Combining this with Corollary 1.8 yields:

Corollary 1.9 (Ambos-Spies et al. 1]). DNR is implied by $\mathrm{WWKL}_{0}$ but not vice versa.

Actually, Corollary 1.8 yields more. For definitions of basic notions from algorithmic randomness and dimension used below, see [5] or [19]. Lutz [16] first studied the effective version of Hausdorff dimension. Later Mayordomo [17] and Ryabko 21] gave a characterization using Kolmogorov complexity. Earlier than them, some results of Levin, Cai, Hartmanis and Staiger also indicated the deep relationship between Hausdorff dimension and Kolmogorov complexity.

Definition 1.10. $\operatorname{dim}(A)=\liminf _{n \rightarrow \infty} \frac{K(A\lceil n)}{n}$.

Clearly, for any constant $0<d \leq 1, T_{d}=\left\{\rho \in 2^{<\omega}: \frac{K_{U}(\rho)}{|\rho|} \geq d\right\}$ is a $\Pi_{1}^{0}$ set that does not admit a computable strong constant-bound-enumeration (the proof proceeds exactly the same as Lemma 5.3); therefore the induced $\Pi_{1}^{0}$ class satisfies the conditions given in Theorem 1.2 .

The following corollary related to algorithmic randomness theory answers a question of Joe Miller.

Corollary 1.11. There exists a DNR function that does not compute any binary sequence with positive effective Hausdorff dimension.

Another interesting application in algorithmic randomness theory is the following.

Corollary 1.12 (Kjos-Hanssen [13]). For every Martin-Löf random set $A \in 2^{\omega}$, there exists an infinite set $G \subseteq A \vee G \subseteq \bar{A}$ such that $G$ does not compute any Martin-Löf random set.

In Section 3 we illustrate the basic ideas and demonstrate the vital part of the construction in an informal style for Step 1 and Step $s$, each parallel with the other. The frame of the proof, "tree-forcing", together with a review of Mathias forcing, is given in Subsection 3.2, Definitions that simplify statements and needed notions are given in Section 2, and the basic facts concerned are given in Section 3 . In 
Section 4 we first define some operations used in the construction which are basic processes in the construction, then in Subsection 4.2 give a concrete construction stated via operations introduced in Subsection 4.1, and in Subsection 4.3 prove that the construction does provide the desired set. Section 5 gives some applications of this result. Section [6 briefly discusses generalizations of this result and proposes related questions.

\section{Preliminaries}

$\Psi$ is sometimes not a single Turing functional but a pair of Turing functionals, $\left\langle\Psi_{l}, \Psi_{r}\right\rangle . \quad \vec{\Psi}$ denotes an array of pairs of Turing functionals. $\vec{\Psi}_{i}$ denotes the $i^{\text {th }}$ component. Similarly $\rho$ is sometimes not a single string but a pair, $\vec{\rho}$ is a sequence of pairs, and $\overrightarrow{\rho_{i}}=\left\langle\rho_{i l}, \rho_{i r}\right\rangle . \quad \Psi^{\rho}$ means $\left\langle\Psi_{l}^{\rho_{l}}, \Psi_{r}^{\rho_{r}}\right\rangle$ and $\vec{\Psi}^{\vec{\rho}}$ means $\left(\left\langle\Psi_{1 l}^{\rho_{1 l}}, \Psi_{1 r}^{\rho_{1 r}}\right\rangle,\left\langle\Psi_{2 l}^{\rho_{2 l}}, \Psi_{2 r}^{\rho_{2 r}}\right\rangle, \ldots\right)$.

$V$ denotes a clopen set of $2^{\omega}$. We equate $V$ with a finite set of strings, i.e. $[V] \preceq=\bigcup_{i=1}^{n}\left[\rho_{i}\right]^{\preceq}$, where $\rho_{1}, \rho_{2}, \ldots, \rho_{n}$ are mutually incompatible. Write height $(V)$ for $\max \{|\rho|: \rho \in V\}$. For a closed set $\mathcal{Q}$ (of $2^{\omega}$ ) identify $\overline{\mathcal{Q}}$ with $\{\rho:[\rho] \subseteq \overline{\mathcal{Q}}\}$ so that $\rho \in \overline{\mathcal{Q}}$ makes sense.

Let $\operatorname{set}(\rho)$ denote $\{i \in \omega: \rho(i)=1\}$, and let $\rho / \sigma(Z / \sigma)$ be $\rho(Z)$ with the first $|\sigma|$ many values replaced by $\sigma$.

For a set $X$, view $X$ as an infinite binary string, and let $\pi_{i}^{n}(X)=X_{i}$ where $X_{i} \in 2^{\omega}$ is such that $X_{i}(j)=X((j-1) n+i)$, i.e. $X=\bigoplus_{i=1}^{n} X_{i}$. $\pi$ can be defined on finite strings in the same way.

We say $X$ codes an ordered $k$-partition of $W$ iff $\bigcup_{i=1}^{k} \pi_{i}^{k}(X)=W$, and say that $\pi_{i}^{k}(X)$ is the $i^{\text {th }}$ part of this partition. A tree $T \subseteq 2^{<\omega}$ is an ordered $k$-partition tree of $W$ iff $\forall X \in[T] X$ is an ordered $k$-partition of $W$. Note that in this paper, "partition" does not mean a piecewise disjoint partition.

Definition 2.1. For $i=1,2, \ldots, u$, let $\mathcal{K}_{i}=\left\{K_{i, 1}, K_{i, 2}, \ldots, K_{i, m_{i}}\right\}$, where each $K_{i, j}$ is a subset of $\{1,2, \ldots, n\}$, and let $\overrightarrow{\mathcal{K}}=\left(\mathcal{K}_{1}, \mathcal{K}_{2}, \ldots, \mathcal{K}_{u}\right)$. We call $\overrightarrow{\mathcal{K}}$ a $u$ supporter of $\{1,2, \ldots, n\}$ iff for every ordered $u$-partition (not necessarily pairwise disjoint) of $\{1,2, \ldots, n\}$, namely $P^{(1)} \cup P^{(2)} \cup \cdots \cup P^{(u)}=\{1,2, \ldots, n\}$, there exists some $\mathcal{K}_{i}$ and some $K_{i, j} \in \mathcal{K}_{i}$ such that $K_{i, j} \subseteq P^{(i)}$.

A sequence of $n$ clopen sets $V^{(1)}, V^{(2)}, \ldots, V^{(n)}$ is $u$-disperse iff for any ordered $u$ partition (not necessarily pairwise disjoint) of $\{1,2, \ldots, n\}, P^{(1)} \cup P^{(2)} \cup \cdots \cup P^{(u)}=$ $\{1,2, \ldots, n\}$, there exists $i \leq u$ such that $\bigcap_{j \in P^{(i)}}\left[V^{(j)}\right] \preceq=\emptyset$.

Definition 2.2. For $n$ many ordered $u$-partitions $X^{(1)}, \ldots, X^{(n)}$ and for $\overrightarrow{\mathcal{K}}=$ $\left\{\mathcal{K}_{1}, \mathcal{K}_{2}, \ldots, \mathcal{K}_{u}\right\}$, where each $\mathcal{K}_{i}$ is a finite class of finite subsets of $\{1,2, \ldots, n\}$ whose members are denoted by $K_{i, j}$, let

$$
\begin{aligned}
\operatorname{Cross}\left(X^{(1)}, X^{(2)}, \ldots, X^{(n)} ; \overrightarrow{\mathcal{K}}\right)=Y \\
=\left(\bigoplus_{1 \leq j \leq\left|\mathcal{K}_{1}\right|} Y_{K_{1, j}}\right) \oplus\left(\bigoplus_{1 \leq j \leq\left|\mathcal{K}_{2}\right|} Y_{K_{2, j}}\right) \oplus \cdots \oplus\left(\bigoplus_{1 \leq j \leq\left|\mathcal{K}_{u}\right|} Y_{K_{u, j}}\right),
\end{aligned}
$$

where $Y_{K_{i, j}}=\bigcap_{p \in K_{i, j} \in \mathcal{K}_{i}} \pi_{i}^{u}\left(X^{(p)}\right)$; i.e. $Y_{K_{i, j}}$ is the intersection of the $i^{\text {th }}$ parts of those $u$-partitions $X^{(p)}$ for which $p \in K_{i, j}$. The order of each $Y_{K_{i, j}}$ in $Y$ "does not matter"; the point is that given $Y, i, j$ and $\overrightarrow{\mathcal{K}}$ one could uniformly (in $Y, i, j$ and $\overrightarrow{\mathcal{K}}$ ) compute each $Y_{K_{i, j}}$. 
If each $\mathcal{K}_{i}$ consists of all of the $m$-element subsets of $\{1,2, \ldots, n\}$, we also abbreviate $\operatorname{Cross}\left(X^{(1)}, X^{(2)}, \ldots, X^{(n)} ; \overrightarrow{\mathcal{K}}\right)$ by $\operatorname{Cross}\left(X^{(1)}, X^{(2)}, \ldots, X^{(n)} ; m\right)$. For $n$ classes $\mathcal{C}^{(1)}, \mathcal{C}^{(2)}, \ldots, \mathcal{C}^{(n)}$ of ordered $u$-partitions,

$$
\begin{aligned}
\operatorname{Cross}\left(\mathcal{C}^{(1)}, \mathcal{C}^{(2)}, \ldots, \mathcal{C}^{(n)} ; \overrightarrow{\mathcal{K}}\right)=\left\{Y \in 2^{\omega}: \exists X^{(i)} \in \mathcal{C}^{(i)} \text { for } 1 \leq i \leq n,\right. \\
\left.Y=\operatorname{Cross}\left(X^{(1)}, \ldots, X^{(n)} ; \overrightarrow{\mathcal{K}}\right)\right\} .
\end{aligned}
$$

Note that the operation Cross can be defined on binary strings in a natural way; therefore if $T^{(1)}, \ldots, T^{(n)}$ are computable trees, then $\operatorname{Cross}\left(\left[T^{(1)}\right], \ldots,\left[T^{(n)}\right] ; \overrightarrow{\mathcal{K}}\right)$ is a $\Pi_{1}^{0}$ class. Let $\operatorname{Cross}\left(T^{(1)}, \ldots, T^{(n)} ; \overrightarrow{\mathcal{K}}\right)$ denote the corresponding computable tree.

Fixing a bijection from $\omega$ to representations of finite subsets of $2^{<\omega}$, for any $X$, we view each function $\Psi_{e}^{X}$ as enumeration, identifying $\Psi_{e}(n)$ with the finite subset of $2^{<\omega}$ it represents, so that $\left|\Psi_{e}(n)\right|, \Psi_{e}(n) \cap X,\left[\Psi_{e}(n)\right] \preceq=\bigcup_{i=1}^{\left|\Psi_{e}(n)\right|}\left[\rho_{i}\right] \preceq$, etc. make sense (if $\Psi_{e}(n) \uparrow$ we view $\Psi_{e}(n)$ as the empty set).

\section{BASIC IDEAS AND SOME STEPS}

We will construct a set $G$ with $G \subseteq A \vee G \subseteq \bar{A}$, satisfying for all $e, j \in \mathbb{N}$ :

- $R(e ; j): \Psi_{e}^{C^{(j)} \oplus G}$ is not a strong $e$-enumeration of $Q$. I.e. $\Psi_{e}^{C^{(j)} \oplus G}$ is not total, or $(\exists n)\left(\left|\Psi_{e}^{C^{(j)} \oplus G}(n)\right|>e \vee \Psi_{e}^{C^{(j)} \oplus G}(n) \cap Q=\emptyset\right)$, or $(\exists l)(\forall n)(\exists \rho \in$ $\left.\Psi_{e}^{C^{(j)}} \oplus G(n)\right)|\rho|<l$.

- $P_{e}:|G| \geq e$.

\subsection{Step 1: Satisfying $R(1 ; j)$.}

Case i. If we can find some $\rho \in 2^{<\omega}$ such that $\operatorname{set}(\rho) \subset A \vee \operatorname{set}(\rho) \subset \bar{A}$ and some $n$ such that $\Psi_{1}^{C^{(j)} \oplus \rho}(n) \downarrow \cap Q=\emptyset \vee\left|\Psi_{1}^{C^{(j)} \oplus \rho}(n)\right|>1$, then we can satisfy $R(1 ; j)$ by finitely extending our initial segment requirement to $\rho$. In this case we say Case $\mathrm{i}$ occurs to $R(1 ; j)$.

Case ii. If there is no such $\rho$, i.e. Case i doesn't occur to $R(1 ; j)$. Then we make $\Psi_{1}^{C^{(j)} \oplus G}$ non-total; we can achieve this by trying to find three disjoint clopen sets $\left[V^{(1)}\right] \preceq,\left[V^{(2)}\right] \preceq,\left[V^{(3)}\right] \preceq$ such that the following classes are non-empty:

$$
\begin{aligned}
{\left[T_{V^{(i)}}\right]=\left\{X=X_{l} \oplus X_{r}: X_{l} \cup X_{r}=\omega \wedge\right.} & {\left[\left(\forall Z \text { s.t. } Z \subseteq X_{l} \vee Z \subseteq X_{r}\right)(\forall n)\right.} \\
& \left.\left.\Psi_{1}^{C^{(j)} \oplus Z}(n) \downarrow \rightarrow\left(\left|\Psi_{1}^{C^{(j)} \oplus Z}(n)\right| \leq 1 \wedge\left[\Psi_{1}^{C^{(j)} \oplus Z}(n)\right] \preceq \cap\left[V^{(i)}\right] \preceq \neq \emptyset\right)\right]\right\} .
\end{aligned}
$$

Let

$$
\left[T_{1}\right]=\operatorname{Cross}\left(\left[T_{V^{(1)}}\right],\left[T_{V^{(2)}}\right],\left[T_{V^{(3)}}\right] ; 2\right),
$$

i.e. $\forall X \in\left[T_{1}\right], X=Y_{1} \oplus Y_{2} \oplus Y_{3} \oplus Y_{4} \oplus Y_{5} \oplus Y_{6}$, where

$$
\begin{array}{llll}
Y_{1}=\pi_{l}^{2}\left(X^{\prime}\right) \cap \pi_{l}^{2}\left(X^{\prime \prime}\right) & \text { for some } & X^{\prime} \in\left[T_{V^{(1)}}\right], X^{\prime \prime} \in\left[T_{V^{(2)}}\right], \\
Y_{2}=\pi_{l}^{2}\left(X^{\prime}\right) \cap \pi_{l}^{2}\left(X^{\prime \prime}\right) & \text { for some } & X^{\prime} \in\left[T_{V^{(2)}}\right], X^{\prime \prime} \in\left[T_{V^{(3)}}\right], \\
Y_{3}=\pi_{l}^{2}\left(X^{\prime}\right) \cap \pi_{l}^{2}\left(X^{\prime \prime}\right) & \text { for some } & X^{\prime} \in\left[T_{V^{(3)}}\right], X^{\prime \prime} \in\left[T_{V^{(1)}}\right], \\
Y_{4}=\pi_{r}^{2}\left(X^{\prime}\right) \cap \pi_{r}^{2}\left(X^{\prime \prime}\right) & \text { for some } & X^{\prime} \in\left[T_{V^{(1)}}\right], X^{\prime \prime} \in\left[T_{V^{(2)}}\right], \\
Y_{5}=\pi_{r}^{2}\left(X^{\prime}\right) \cap \pi_{r}^{2}\left(X^{\prime \prime}\right) & \text { for some } & X^{\prime} \in\left[T_{V^{(2)}}\right], X^{\prime \prime} \in\left[T_{V^{(3)}}\right], \\
Y_{6}=\pi_{r}^{2}\left(X^{\prime}\right) \cap \pi_{r}^{2}\left(X^{\prime \prime}\right) & \text { for some } & X^{\prime} \in\left[T_{V^{(3)}}\right], X^{\prime \prime} \in\left[T_{V^{(1)}}\right] .
\end{array}
$$


Note:

(1) $\left[T_{V^{(i)}}\right]$ is a $\Pi_{1}^{0, C^{(j)}}$ class. For any general $\Psi$ (instead of $\left.\Psi_{1}\right)$ and $V$ (instead of $V^{(i)}$ ) the index of $\left[T_{V}\right]$, which is induced by $\Psi, V$, can be uniformly computed from indices for $\Psi$ and $V$.

(2) If $[V] \preceq \supseteq[Q]$, then $T_{V} \neq \emptyset$, as otherwise Case i occurs: For $X_{1}=A$ and $X_{2}=\bar{A}$, if we assume that $X_{1} \oplus X_{2} \notin T_{V}$, then there are a $\rho$ such that $\operatorname{set}(\rho) \subseteq A \vee \operatorname{set}(\rho) \subseteq \bar{A}$ and an $n \in \mathbb{N}$ with $\left[\Psi_{1}^{C^{(j)} \oplus \rho}(n) \downarrow\right] \preceq \cap[V] \preceq=\emptyset \vee$ $\left|\Psi_{1}^{C^{(j)} \oplus \rho}(n)\right|>1$, which implies that $\left[\Psi_{1}^{C^{(j)} \oplus \rho}(n)\right] \preceq \cap Q=\emptyset \vee\left|\Psi_{1}^{C^{(j)} \oplus \rho}(n)\right|>$ 1.

(3) For any $i \leq 6, G \subseteq Y_{i}, \Psi_{1}^{C^{(j)} \oplus G}$ is not a strong 1-enumeration of $Q$. To see this, suppose on the contrary that for some $G \subseteq Y_{1}, \Psi_{1}^{C^{(j)} \oplus G}$ is a strong 1-enumeration of $Q$. Then there exists $n$ such that $\forall \rho \in \Psi_{1}^{C^{(j)}} \oplus G(n),|\rho|=$ $n>\max \left\{\operatorname{height}\left(V^{(1)}\right)\right.$, height $\left.\left(V^{(2)}\right)\right\}$. Then $\forall \rho \in \Psi_{1}^{C^{(j)} \oplus G}(n)$ either $[\rho] \preceq \cap$ $\left[V^{(1)}\right] \preceq=\emptyset$ or $[\rho] \preceq \cap\left[V^{(2)}\right] \preceq=\emptyset$ (since $\left[V^{(1)}\right],\left[V^{(2)}\right]$ are disjoint), say $[\rho] \preceq \cap\left[V^{(1)}\right] \preceq=\emptyset$, which implies $\left[\Psi_{1}^{C^{(j)} \oplus G}(n)\right] \cap\left[V^{(1)}\right] \preceq=\emptyset$ (note that at this step there is at most one $\left.\rho \in \Psi_{1}^{C^{(j)} \oplus G}(n)\right)$. But $G \subseteq Y_{1} \subseteq \pi_{l}^{2}\left(X^{\prime}\right)$ for some $X^{\prime} \in\left[T_{V^{(1)}}\right]$, and by the definition of $\left[T_{V^{(1)}}\right], \forall n,\left[\Psi_{1}^{C^{(j)} \oplus G}(n)\right] \preceq \cap$ $\left[V^{(1)}\right] \preceq \neq \emptyset$ if $\Psi_{1}^{C^{(j)} \oplus G}(n) \downarrow$.

(4) $\left[T_{1}\right]$ is a $\Pi_{1}^{0, C^{(j)}}$ class (note that Cross can be applied to binary strings) whose index can be $C^{(j)}$-computed from indices for $\Psi, V^{(1)}, V^{(2)}$, and $V^{(3)}$.

(5) $\bigcup_{i=1}^{6} Y_{i}=\omega$ (see Fact 3.6 this is just the pigeonhole principle). This is why we try to choose three mutually disjoint clopen sets at this step; i.e. if we choose, say, two disjoint clopen sets, then the union of all parts of some path through $T_{1}$ might be finite, which leads to difficulties at the next step of the construction. The advantage of requiring $T$ to be an ordered-partitiontree will be clear in Lemma 4.5. In general, such three mutually disjoint clopen sets can't be found, so instead we try to find a 2-disperse sequence of clopen sets for some 2-supporter at this step, while at later steps 2-disperse sequences will not be enough and will have to be replaced by $k^{\prime}$-disperse sequences.

How the requirement $R(1 ; j)$ is satisfied: If Case i occurs, we require that if finally $G \subseteq A(\bar{A}) \wedge \operatorname{set}(\rho) \subset A(\bar{A})$, then $G \supset \rho$. If Case ii occurs, we require that for some path $X \in\left[T_{1}\right], G$ will be contained in some $Y_{i}$ such that $Y_{i}=\pi_{i}^{6}(X)$. By the above notes clearly $R(1 ; j)$ is satisfied. Furthermore, Lemma 4.6 will prove that if no such 2-disperse sequence exists such that all induced classes are non-empty (i.e. Case ii does not occur), then Case i occurs; otherwise one can compute a strong $k$-enumeration of $Q$.

3.2. Tree forcing. A Mathias forcing condition is a pair $(\rho, L)$ where $\rho \in 2^{<\omega}$ and $L \in 2^{\omega}$. We write $(\rho, L) \geq\left(\rho^{\prime}, L^{\prime}\right)$ and say that $\left(\rho^{\prime}, L^{\prime}\right)$ extends $(\rho, L)$ iff $\rho^{\prime} \supset \rho \wedge L^{\prime} \cup \operatorname{set}\left(\rho^{\prime}\right) \subseteq L \cup \operatorname{set}(\rho)$. A set $G$ satisfies $(\rho, L)$ iff $G \supset \rho \wedge G \subseteq \operatorname{set}(\rho) \cup L$.

In this paper, a tree forcing condition is a triple $(\vec{\rho}, T, k)$, where $T \subseteq 2^{<\omega}$ is a tree and $\vec{\rho}=\left(\left\langle\rho_{1 l}, \rho_{1 r}\right\rangle,\left\langle\rho_{2 l}, \rho_{2 r}\right\rangle, \ldots,\left\langle\rho_{k l}, \rho_{k r}\right\rangle\right)$ is an array of $k$ pairs of binary strings, 
such that:

(1) each $X \in[T]$ together with $\vec{\rho}$ codes in a uniform way $2 k$ Mathias forcing conditions, $\left\langle\rho_{1 l}, \pi_{1}^{k}(X)\right\rangle,\left\langle\rho_{1 r}, \pi_{1}^{k}(X)\right\rangle, \ldots,\left\langle\rho_{k l}, \pi_{k}^{k}(X)\right\rangle,\left\langle\rho_{k r}, \pi_{k}^{k}(X)\right\rangle$, i.e. there is one initial segment requirement pair for each part.

(2) $\bigcup_{i=1}^{k} \pi_{i}^{k}(X)={ }^{*} \omega$ (i.e. $\bigcup_{i=1}^{k} \pi_{i}^{k}(X)$ is $\omega$ minus some finite set).

(3) $(\forall i \leq k, X \in[T]) \pi_{i}^{k}(X) \cap\left\{1,2, \ldots, \max \left\{\left|\rho_{i l}\right|,\left|\rho_{i r}\right|\right\}\right\}=\emptyset$.

(4) $\operatorname{set}\left(\rho_{i l}\right) \subseteq A \wedge \operatorname{set}\left(\rho_{i r}\right) \subseteq \bar{A}$, where $A \in 2^{\omega}$ is the given set in Theorem 1.2

A set $G$ satisfies $(\vec{\rho}, T, k)$ iff there exist $X \in[T]$ and $i \leq k$ such that $G$ satisfies either $\left\langle\rho_{i l}, \pi_{i}^{k}(X)\right\rangle$ or $\left\langle\rho_{i r}, \pi_{i}^{k}(X)\right\rangle$. $\left(\vec{\rho}^{\prime}, T^{\prime}, k^{\prime}\right)$ extends $(\vec{\rho}, T, k)$, written as $\left(\vec{\rho}^{\prime}, T^{\prime}, k^{\prime}\right) \leq(\vec{\rho}, T, k)$, iff there exists a function $p:\left\{1,2, \ldots, k^{\prime}\right\} \rightarrow\{1,2, \ldots, k\}$ such that

(1) $\forall i \leq k^{\prime}, \rho_{i l}^{\prime} \supset \rho_{p(i), l} \wedge \rho_{i r}^{\prime} \supset \rho_{p(i), r}$,

(2) $\forall i \leq k^{\prime}, \forall X^{\prime} \in\left[T^{\prime}\right] \exists X \in[T], \operatorname{set}\left(\rho_{i l}^{\prime}\right) \cup \pi_{i}^{k^{\prime}}\left(X^{\prime}\right) \subseteq \operatorname{set}\left(\rho_{p(i), l}\right) \cup \pi_{p(i)}^{k}(X)$ and similarly for the right-hand side. We call part $i$ of $T^{\prime}$ a child part of part $p(i)$ of $T$.

We will construct a sequence $\left(\vec{\rho}^{1}, T_{1}, k_{1}\right) \geq\left(\vec{\rho}^{2}, T_{2}, k_{2}\right) \geq \cdots \geq\left(\vec{\rho}^{s}, T_{s}, k_{s}\right) \geq$ ‥ of tree forcing conditions. Each part $i \leq k_{s}$ of $\left(\vec{\rho}^{s}, T_{s}, k_{s}\right)$ will correspond to a progress information sequence $\left(j ; e_{i, r}^{s, j}, e_{i, l}^{s, j}\right)$ for $j \in \mathbb{N}$, which means that for all $i \leq k_{s}$ and $X \in\left[T_{s}\right]$, if $G$ satisfies $\left\langle\rho_{i l}^{s}, \pi_{i}^{k_{s}}(X)\right\rangle$, then $G$ satisfies $R(e ; j)$ for all $j \in \mathbb{N}$ and $e<e_{i, l}^{s, j}$, and if $G$ satisfies $\left\langle\rho_{i r}^{s}, \pi_{i}^{k_{s}}(X)\right\rangle$, then $G$ satisfies $R(e ; j)$ for all $j \in \mathbb{N}$ and $e<e_{i, r}^{s, j}$. Furthermore, we will have $\left[T_{s}\right] \neq \emptyset$, and each $T_{s}$ will be computable in some $C \in \mathcal{M}$ where $\mathcal{M}$ is the given countable class in Theorem 1.2.

Let $C^{(j)}$ for $j \in \mathbb{N}$ be a sequence of sets of $\mathcal{M}$ that is cofinal in $\mathcal{M}$, i.e. $(\forall j \in \mathbb{N})\left[C^{(j)} \in \mathcal{M} \wedge\left(\forall C \in \mathcal{M} \exists C^{(j)}, C \leq_{T} C^{(j)}\right)\right]$, and such that $\forall j \in \mathbb{N}, C^{(j)} \leq_{T}$ $C^{(j+1)}$. Fix a sequence $C^{t_{s}}$ for $s \in \mathbb{N}$ of elements of $\left\{C^{(j)}\right\}_{j \in \mathbb{N}}$ such that each $C^{(j)}$ appears infinitely often in this sequence, i.e. $\exists^{\infty} s, t_{s}=j$. Step $s$ will be devoted to $R\left(\cdots ; t_{s}\right)$, which means we make sure that if part $i^{\prime}$ of $T_{s}$ has a child in $T_{s+1}$, then on each child of part $i^{\prime}$, for example part $i$ of $T_{s+1}$, for $j=t_{s}$ either $e_{i r}^{s, j}>e_{i^{\prime} r}^{s-1, j}$ or $e_{i l}^{s, j}>e_{i^{\prime} l}^{s-1, j}$.

To avoid too many indices we use formal parameters $T, \vec{\rho}, \vec{\Psi}$. Their values are updated at each step, so $\vec{\Psi}=\vec{e}^{s-1, j}$ means we assign $e_{i l}^{s-1, j}$ to be the index of $\Psi_{i l}$, and writing $T=\left\{\rho \in 2^{<\omega}: \ldots \sigma \in T \ldots\right\}$ makes sense. Within each step, and from one step to the next, progress information may also need to be updated, so writing $e_{i l}^{s-1, j}=e_{i l}^{s-1, j}+1$ or $e_{i l}^{s, j}=e_{i l}^{s-1, j}+1$ also makes sense.

3.3. Some definitions and facts. Before we get a glimpse at Step $s$, we make the following definition in order to simplify our statements:

\section{Definition 3.1.}

(1) $\Psi^{C \oplus \rho}$ abandons $V$ on a set $Y$ iff there exist $Z \subseteq Y$ and $n \in \mathbb{N}$ such that either $\Psi^{C \oplus Z / \rho}(n) \downarrow=D$ for a finite set $D$ with $[D] \preceq \cap[V] \preceq=\emptyset$, or $\left|\Psi^{C \oplus Z / \rho}(n)\right|$ is greater than the index of $\Psi$.

(2) $\left\langle\Psi_{l}^{C \oplus \rho_{l}}, \Psi_{r}^{C \oplus \rho_{r}}\right\rangle$ (also denoted by $\Psi^{C \oplus \rho}$ ) abandons $V$ on $X_{1} \oplus X_{2}$ iff either $\Psi_{l}^{C \oplus \rho_{l}}$ abandons $V$ on $X_{1}$ or $\Psi_{r}^{C \oplus \rho_{r}}$ abandons $V$ on $X_{2}$. 
(3) $\Psi^{C \oplus \rho}$ (which means $\left\langle\Psi_{l}^{C \oplus \rho_{l}}, \Psi_{r}^{C \oplus \rho_{r}}\right\rangle$ ) abandons $V$ on $X$ iff for all ordered 2-partitions $X_{1} \cup X_{2}=X,\left\langle\Psi_{l}^{C \oplus \rho_{l}}, \Psi_{r}^{C \oplus \rho_{r}}\right\rangle$ abandons $V$ on $X_{1} \oplus X_{2}$. I.e. $\left\langle\Psi_{l}^{C \oplus \rho_{l}}, \Psi_{r}^{C \oplus \rho_{r}}\right\rangle$ doesn't abandon $V$ on $X$ iff there exists an ordered partition $X_{1} \cup X_{2}=X$ such that $\forall Y \supset \rho_{l}, Y \subseteq X_{1} \cup \operatorname{set}\left(\rho_{l}\right) \Rightarrow(\forall n)\left[\left(\Psi_{l}^{C \oplus Y}(n) \uparrow\right)\right.$ $\left.\vee\left(\left[\Psi_{l}^{C \oplus Y}(n)\right] \preceq \cap[V] \preceq \neq \emptyset \wedge\left|\Psi_{l}^{C \oplus Y}(n)\right| \leq l\right)\right]$ and $\forall Y \supset \rho_{r}, Y \subseteq X_{2} \cup$ $\operatorname{set}\left(\rho_{r}\right) \Rightarrow(\forall n)\left[\left(\Psi_{r}^{C \oplus Y}(n) \uparrow\right) \vee\left(\left[\Psi_{r}^{C \oplus Y}(n)\right] \preceq \cap[V] \preceq \neq \emptyset \wedge\left|\Psi_{r}^{C \oplus Y}(n)\right| \leq r\right)\right]$.

(In the above definitions, "abandons on a set" can be replaced by "abandons on a finite string"; the definitions generalize naturally.)

(4) $\vec{\Psi}^{C \oplus \vec{\rho}}$ abandons $V$ on $X=\bigoplus_{i=1}^{k} X_{i}$ iff $\exists i \leq k$ such that the pair $\Psi_{i}^{C \oplus \rho_{i}}$ abandons $V$ on $X_{i}$. I.e. $\vec{\Psi}^{C \oplus \vec{\rho}}$ doesn't abandon $V$ on $X=\bigoplus_{i=1}^{k} X_{i}$ iff for all $i$, there exist $X_{i l}, X_{i r}$ with $X_{i l} \cup X_{i r}=X_{i}$ such that $\Psi_{i l}^{C \oplus \rho_{i l}}$ doesn't abandon $V$ on $X_{i l}$ and $\Psi_{i r}^{C \oplus \rho_{i r}}$ doesn't abandon $V$ on $X_{i r}$.

We also say that $\vec{\Psi}^{C \oplus \vec{\rho}}$ doesn't abandon $V$ on $\bigoplus_{i=1}^{k}\left(X_{i l} \oplus X_{i r}\right)$ to indicate that for all $i, \Psi_{i l}^{C \oplus \rho_{i l}}$ doesn't abandon $V$ on $X_{i l}$ and $\Psi_{i r}^{C \oplus \rho_{i r}}$ doesn't abandon $V$ on $X_{i r}$. (Note that this is a little abuse of notation, but the lower index $i l, i r$ shall avoid confusion.) Let $\pi_{i l}^{k}(X)=X_{i l}$ and $\pi_{i r}^{k}(X)=X_{i r}$.

The following two simple facts illustrate the central idea of the construction.

Fact 3.2. Let $V^{(1)}, V^{(2)}, \ldots, V^{(m)}$ be an $e$-disperse sequence of clopen sets and let $\Psi$ be a Turing functional such that for each $i, \Psi$ doesn't abandon $V^{(i)}$ on $X$. Then, for any $Y \subseteq X, \Psi^{Y}$ is not total or is not a strong e-enumeration. (The proof also holds if $\Psi$ is relativized, i.e. it also holds if for some oracle $C$ and some $\rho$ we replace $\Psi$ by $\Psi^{C \oplus \rho}$ and $\Psi^{Y}$ by $\Psi^{C \oplus Y / \rho}$.)

Proof. Here and below $\Psi$ abandoning $V$ means $\Psi^{\emptyset \oplus \varepsilon}$ abandoning $V$, where $\varepsilon$ is the empty string. Suppose not. Then there is some $n$ and some $Y \subseteq X$ such that $\left|\Psi^{Y}(n)\right| \leq e$ and

$$
\forall \rho \in \Psi^{Y}(n),|\rho|>\max \left\{\operatorname{height}\left(V^{(1)}\right), \operatorname{height}\left(V^{(2)}\right), \ldots, \operatorname{height}\left(V^{(m)}\right)\right\},
$$

and furthermore for each $i \leq m,\left[V^{(i)}\right] \preceq \cap\left[\Psi^{Y}(n)\right] \preceq \neq$. For $1 \leq i \leq e$, let $P^{(i)}=\left\{V^{(j)}:\left[V^{(j)}\right] \preceq \cap\left[\rho_{i}\right] \preceq \neq \emptyset\right.$, where $\rho_{i}$ is the $i^{\text {th }}$ string in $\left.\Psi^{\bar{Y}}(n)\right\}$ (let $P^{(i)}=P^{(1)}$ if $\left.i>\left|\Psi_{e}^{Y}(n)\right|\right)$. Note that since $\left|\rho_{i}\right|>$ height $V^{(r)}$, we have that $\left[\rho_{i}\right]^{\preceq} \cap\left[V^{(r)}\right] \preceq \neq \emptyset$ implies $\left[\rho_{i}\right] \preceq \subseteq\left[V^{(r)}\right] \preceq$. The $P^{(i)}$ form an $e$-partition of $V^{(1)}, V^{(2)}, \ldots, V^{(m)}$, but $\forall i, \bigcap_{r \in P^{(i)}}\left[V^{(r)}\right] \preceq \supseteq\left[\rho_{i}\right] \preceq \neq \emptyset$, which contradicts the assumption that $V^{(1)}, V^{(2)}, \ldots, V^{(m)}$ is an $e$-disperse sequence.

Fact 3.3. If a single Turing functional $\Psi_{e}^{C \oplus \rho}$ doesn't abandon $V$ on $X$, then for any $Y \subseteq X, \Psi_{e}^{C \oplus \rho}$ doesn't abandon $V$ on $Y$.

Proof. By the definition of "abandon", item (1).

\section{Combining Fact 3.2 and Fact 3.3 .}

Fact 3.4. Let $V^{(1)}, V^{(2)}, \ldots, V^{(m)}$ be an $e$-disperse sequence of clopen sets and let $\Psi$ be a Turing functional such that for each $i, \Psi$ as a strong $e$-enumeration doesn't abandon $V^{(i)}$ on a set $X^{(i)}$. Then, for any $Y \subseteq \bigcap_{i=1}^{m} X^{(m)}, \Psi^{Y}$ is not total or is not a strong $e$-enumeration. (As Fact 3.2 this also holds for the relativized version.)

Together with Fact 3.4, the following two facts tell us how to apply the Cross operation in order to ensure that $T_{s}$ is an ordered-partition-tree. 
Fact 3.5. Let $e_{1}, e_{2}, \ldots, e_{u}$ be $u$ many positive numbers and let $k^{\prime}=\sum_{i=1}^{u} e_{i}$. If $V^{(1)}, V^{(2)}, \ldots, V^{(n)}$ is a $k^{\prime}$-disperse sequence of clopen sets, for each $1 \leq i \leq$ $u$, let $\mathcal{K}_{i}=\left\{K \subseteq\{1,2, \ldots, n\}:\left\{V^{(j)}\right\}_{j \in K}\right.$ is an $e_{i}$-disperse class $\}$. Then $\overrightarrow{\mathcal{K}}=$ $\left(\mathcal{K}_{1}, \ldots, \mathcal{K}_{u}\right)$ is a $u$-supporter of $\{1,2, \ldots, n\}$.

Proof. Suppose, on the contrary, there is an ordered partition $P^{(1)}, P^{(2)}, \ldots, P^{(u)}$ of $\{1,2, \ldots, n\}$ such that for all $1 \leq i \leq u, P^{(i)} \notin \mathcal{K}_{i}$, i.e. $\left\{V^{(j)}\right\}_{j \in P^{(i)}}$ is not an $e_{i}$-disperse sequence of clopen sets. Then for each $i$, there exists a partition $P^{(i, 1)} \cup$ $P^{(i, 2)} \cup \cdots \cup P^{\left(i, e_{i}\right)}=P^{(i)}$ such that $\left(\forall e \leq e_{i}\right)\left(\bigcap_{j \in P^{(i, e)}}\left[V^{(j)}\right] \preceq \neq \emptyset\right)$. However, then $P^{(1,1)}, P^{(1,2)}, \ldots, P^{\left(1, e_{1}\right)}, \ldots, P^{(i, j)}, \ldots, P^{\left(K, e_{u}\right)}$ is a $k^{\prime}$-partition of $\{1,2, \ldots, n\}$ that contradicts the assumption that $V^{(1)}, V^{(2)}, \ldots, V^{(n)}$ is a $k^{\prime}$-disperse class of clopen sets.

By the definition of $u$-supporter we have:

Fact 3.6. Let $\overrightarrow{\mathcal{K}}=\left(\mathcal{K}_{1}, \ldots, \mathcal{K}_{u}\right)$ be a $u$-supporter of $\{1,2, \ldots, n\}$, let the sets $X^{(1)}, X^{(2)}, \ldots, X^{(n)}$ be ordered $u$-partitions of $W$, and let $K^{\prime}=\sum_{i=1}^{K}\left|\mathcal{K}_{i}\right|$. Then $\operatorname{Cross}\left(X^{(1)}, X^{(2)}, \ldots, X^{(n)} ; \overrightarrow{\mathcal{K}}\right)$ is an ordered $K^{\prime}$-partition of $W$.

Proof. This is straightforward by the definition of $u$-supporter. We show that for each $x \in W$ there exists an $i$ and $K_{i, j} \in \mathcal{K}_{i}$, such that $x \in \bigcap_{p \in K_{i, j}} \pi_{i}^{u}\left(X^{(p)}\right)$. Note that for each $m \leq n, x$ belongs to some part of $X^{(m)}$, and therefore $P^{(i)}=\{m \leq$ $\left.n: x \in \pi_{i}^{u}\left(X^{(m)}\right)\right\}, 1 \leq i \leq u$, is a $u$-partition of $\{1,2, \ldots, n\}$. By the definition of $u$-supporter, there exists an $i$ and $K_{i, j} \in \mathcal{K}_{i}$ such that $K_{i, j} \subseteq P^{(i)}$, which implies that $x \in \bigcap_{p \in K_{i, j}} \pi_{i}^{K}\left(X^{(p)}\right)$.

Furthermore, the following plain fact tells us what kind of finite extension of an initial segment requirement is allowed.

Fact 3.7. If $\Psi^{C \oplus \rho}$ doesn't abandon $V$ on $Y$ and $\sigma \supset \rho$ is such that $\operatorname{set}(\sigma) \subseteq$ $Y \cup \operatorname{set}(\rho)$, then $\Psi^{C \oplus \sigma}$ also doesn't abandon $V$ on $Y$. Furthermore, if $\vec{\Psi}^{C \oplus \vec{\rho}}$ doesn't abandon $V$ on $X=\bigoplus_{i=1}^{k} X_{k}$ and $\vec{\sigma} \supset \vec{\rho}$ is such that $(\forall i \leq k) \operatorname{set}\left(\sigma_{i}\right) \subseteq X_{i} \cup \operatorname{set}\left(\rho_{i}\right)$, then $\vec{\Psi}^{C \oplus \vec{\sigma}}$ also doesn't abandon $V$ on $X$.

3.4. Step s: Construct $\left(\vec{\rho}^{s}, T_{s}\right)$. Suppose we have a $C$-computable ordered $k_{s-1^{-}}$ partition tree $T_{s-1}$, for some $C \in \mathcal{M}$ (here we mean a $k$-partition of some $W={ }^{*} \omega$; note that in above argument $T_{1}$ is a 6 -partition tree of $\omega$ ) and we need to construct $T_{s}$ ensuring that on each child part, either the left-hand side or the right-hand side steps forward; i.e. if $G$ satisfies $\left(\rho_{i l}, \pi_{i}^{k}(X)\right)$ for some $X \in\left[T_{s-1}\right]$, then $\Psi_{i l}^{C^{(j)} \oplus G}$ is not a strong $e_{i, l}^{s-1, j}$-enumeration of $Q$ (where $e_{i, l}^{s-1, j}$ is the index of $\Psi_{i l}$ as updated at step $s-1)$, and if $G$ satisfies $\left(\rho_{i r}, \pi_{i}^{k}(X)\right)$ for some $X \in\left[T_{s-1}\right]$, then $\Psi_{i r}^{C^{(j)} \oplus G}$ is not a strong $e_{i, r}^{s-1, j}$-enumeration of $Q$.

Case i. If there exists $\rho$ and $i$ satisfying either

$$
\begin{aligned}
\rho \supset \rho_{i l} \wedge\left(\exists n,\left|\Psi_{i l}^{C^{(j)} \oplus \rho}(n)\right|>\right. & \left.e_{i l}^{s-1, j} \vee\left[\Psi_{i l}^{C^{(j)} \oplus \rho}(n) \downarrow\right] \cap[Q]=\emptyset\right) \\
& \wedge(\operatorname{set}(\rho) \subseteq A) \wedge\left(\exists X \in[T], \operatorname{set}(\rho) \subseteq \pi_{i}^{k}(X) / \rho_{i l}\right)
\end{aligned}
$$


or

$$
\begin{aligned}
\rho \supset \rho_{i r} \wedge\left(\exists n,\left|\Psi_{i r}^{C^{(j)} \oplus \rho}(n)\right|\right. & \left.>e_{i r}^{s-1, j} \vee\left[\Psi_{i r}^{C^{(j)} \oplus \rho}(n) \downarrow\right] \cap[Q]=\emptyset\right) \\
& \wedge(\operatorname{set}(\rho) \subseteq \bar{A}) \wedge\left(\exists X \in[T], \operatorname{set}(\rho) \subseteq \pi_{i}^{k}(X) / \rho_{i r}\right),
\end{aligned}
$$

then we can extend $\rho_{i l}$ or $\rho_{i r}$ to $\rho$, which ensures that either $\Psi_{i l}^{C^{(j)} \oplus G}$ or $\Psi_{i r}^{C^{(j)} \oplus G}$ is not a strong constant-bound-enumeration of $Q$, assuming $G$ satisfies $\left\langle\rho_{i l}, \pi_{i}^{k}(X)\right\rangle$ or $\left\langle\rho_{i r}, \pi_{i}^{k}(X)\right\rangle$, respectively, for some $X \in[T]$. Note that this operation doesn't increase the total number of parts and that the new $\Pi_{1}^{0}$ class $[T]$ is still non-empty since $\exists X \in[T]$ such that $\operatorname{set}(\rho) \subseteq \pi_{i}^{k}(X) / \rho_{i l}$ (or similarly for the right-hand side). During the construction the above process will be repeatedly carried out within Step $s$ until Case i fails to occur, which must happen as proved in Lemma 4.4

Case ii. If Case i fails to occur. Let $k^{\prime}=\sum_{i=1}^{k}\left(e_{i, l}^{s-1, j}+e_{i, r}^{s-1, j}\right)$, where $k=k_{s-1}$, and each $e_{i, r}^{s-1, j}$ is as updated in previous applications of Case i this stage, if any, i.e. the present progress for $R(\cdots ; j)$, part $i$. Try to find a $k^{\prime}$-disperse sequence of clopen sets $V^{(1)}, V^{(2)}, \ldots, V^{(n)}$ (in Step $1, k^{\prime}=1+1=2$ ) such that the following classes are non-empty for $m \leq n$ :

$$
\begin{aligned}
{\left[T_{V^{(m)}}\right]=\left\{X=\bigoplus_{i=1}^{k}\left(X_{i l}\right.\right.} & \left.\oplus X_{i r}\right): \bigoplus_{i=1}^{k}\left(X_{i l} \cup X_{i r}\right) \in\left[T_{s}\right] \\
& \left.\wedge \vec{\Psi}^{C^{(j)} \oplus \vec{\rho}} \text { doesn't abandon } V^{(m)} \text { on } \bigoplus_{i=1}^{k}\left(X_{i l} \oplus X_{i r}\right)\right\} .
\end{aligned}
$$

The above condition means that

$$
\begin{aligned}
\left(\forall i \leq k \forall G \subseteq X_{i l} \forall n\right)(\mid & \Psi_{i l}^{C^{(j)} \oplus G / \rho_{i l}}(n) \mid \leq e_{i, l}^{s-1, j} \\
& \left.\wedge\left(\Psi_{i l}^{C^{(j)} \oplus G / \rho_{i l}}(n) \uparrow \vee\left[\Psi_{i l}^{C^{(j)} \oplus G / \rho_{i l}}(n)\right] \preceq \cap\left[V^{(m)}\right] \preceq \neq \emptyset\right)\right),
\end{aligned}
$$

and similarly for the right-hand side. Note that each $\left[T_{V^{(m)}}\right]$ is a $\Pi_{1}^{0, C^{(j)}}$ class of ordered $2 k$-partitions.

For all $1 \leq i \leq 2 k$, if $i$ is even, then let $i^{\prime}=\frac{i}{2}$ and define $\mathcal{K}_{i}=\{K \subseteq\{1,2, \ldots, n\}$ : $\left\{V^{(m)}\right\}_{m \in K}$ is an $e_{i^{\prime}, l}^{s-1, j}$-disperse class $\}$; if $i$ is odd, then let $i^{\prime}=\frac{i+1}{2}$ and define $\mathcal{K}_{i}=\left\{K \subseteq\{1,2, \ldots, n\}:\left\{V^{(m)}\right\}_{m \in K}\right.$ is an $e_{i^{\prime}, r}^{s-1, j}$-disperse class $\}$. Note that by Fact 3.5, $\overrightarrow{\mathcal{K}}=\left(\mathcal{K}_{1}, \ldots, \mathcal{K}_{2 k}\right)$ is a $2 k$-supporter of $\{1,2, \ldots, n\}$.

Let

$$
T_{s}=\operatorname{Cross}\left(T_{V^{(1)}}, T_{V^{(2)}}, \ldots, T_{V^{(n)}} ; \overrightarrow{\mathcal{K}}\right)
$$

i.e.

where

$$
\forall X \in\left[T_{s}\right], X=\bigoplus_{i \leq 2 k}\left(\bigoplus_{j \leq\left|\mathcal{K}_{i}\right|} Y_{K_{i, j}}\right)
$$

$$
Y_{K_{i, j}}=\bigcap_{p \in K_{i, j}} \pi_{i}^{2 k}\left(X^{(p)}\right) \text { for } X^{(p)} \in\left[T_{V^{(p)}}\right] .
$$

Then we try to satisfy positive requirements by appropriately extending the present initial segment requirements which were updated in previous applications of Case i of the P-Operation in the last stage. Then we update progress information (i.e. update $\vec{e}^{j}$ ). 
Note:

(1) $\left[T_{V^{(m)}}\right]$ is a $\Pi_{1}^{0, C \oplus C^{(j)}}$ class.

(2) If $[V] \preceq \supseteq[Q]$, then $\left[T_{V}\right] \neq \emptyset$, as otherwise Case i occurs.

(3) For all $i$, if $i$ is even, then $\Psi_{i l}^{C^{(j)} \oplus G / \rho_{i l}}$ is not a strong $e_{i l}^{s-1, j}$-enumeration of $Q$ for any $G \subseteq Y_{K_{i, j}}$, and if $i$ is odd, then $\Psi_{i r}^{C^{(j)} \oplus G / \rho_{i r}}$ is not a strong $e_{i r}^{s-1, j}$-enumeration of $Q$ for any $G \subseteq Y_{K_{i, j}}$ (by Fact 3.4 and the definition of $\overrightarrow{\mathcal{K}})$; i.e. on each child part $R(\cdots ; j)$ steps forward on either the left-hand side or the right-hand side.

(4) $\left[T_{s}\right]$ is a $\Pi_{1}^{0, C \oplus C^{(j)}}$ class.

(5) $\bigcup_{i \leq 2 k}\left(\bigcup_{j \leq\left|\mathcal{K}_{i}\right|} Y_{K_{i, j}}\right)=W$; i.e. $T_{s}$ is an ordered- $K^{\prime}$-partition tree, where $K^{\prime}=\sum_{i=1}^{K}\left|\mathcal{K}_{i}\right|$. (See Fact 3.6, this is why we choose a $k^{\prime}$-disperse class of clopen sets at this step.)

How the requirement $R(e ; j)$ is satisfied: For some path $X \in\left[T_{s}\right]$, $G$ will be contained in some $\pi_{i^{\prime}}^{K^{\prime}}(X)$, and we will have $G \supset \rho_{i^{\prime} l}$ or $G \supset \rho_{i^{\prime} r}$. Therefore either $R\left(e_{i l}^{s-1, j} ; j\right)$ or $R\left(e_{i r}^{s-1, j} ; j\right)$ is satisfied on part $i^{\prime}$ of $T_{s}$, where part $i$ of $T_{s-1}$ is the parent of part $i^{\prime}$ of $T_{s}$.

\section{Construction And verification}

Given a countable class $\mathcal{M}$ closed under disjoint union, a set $A$, and a closed set $[Q]$ of $2^{\omega}$ satisfying the conditions stated in Theorem 1.2 , we give a concrete construction of an infinite set $G$ with $G \subseteq A \vee G \subseteq \bar{A}$ such that $\forall C \in \mathcal{M}, G \oplus$ $C$ does not compute a constant-bound-enumeration of $Q$, and we prove that the construction provides the desired $G$.

If $A \leq_{T} C^{(j)}$ for some $C^{(j)} \in \mathcal{M}$, then just let $G=A$ if $A$ is infinite, and $G=\bar{A}$ otherwise. So without loss of generality assume $\forall C \in \mathcal{M}, A \not{ }_{T} C$.

4.1. Some operations. Suppose we are given $A \in 2^{\omega}$, and let $T$ be an ordered $k$-partition tree of $2^{<\omega}$, with $\vec{\rho}$ being the corresponding initial segment requirement.

To satisfy positive requirements we will apply the P-Operation.

Definition 4.1 (P-Operation). The P-Operation applied to the left-hand side of $T$ 's part $i$ : Choose $\rho \supset \rho_{i l}$ (if one exists) such that $\left[T_{\rho, i}\right]=\left\{X \in[T]: \operatorname{set}\left(\pi_{i}^{k}(X)\right) \supset\right.$ $\operatorname{set}(\rho)\} \neq \emptyset$ and $\emptyset \neq \operatorname{set}(\rho)-\operatorname{set}\left(\rho_{i l}\right) \subset A$, and then update initial segments: let $\rho_{i l}=\rho / \rho_{i l}$, let $\rho_{j l}=\rho_{j l}$ for $j \neq i$, and let $\rho_{i r}=\rho_{i r}$ for all $i$. Finally let $T=T_{\rho, i}$.

If such a $\rho$ exists, we say the P-Operation succeeds. Otherwise do nothing; in this case we say the P-Operation fails.

The P-Operation applied to the right-hand side of $T$ 's part $i$ is analogous, with $A$ replaced by $\bar{A}$.

To satisfy $R(\cdots ; j)$ we will apply the R-i-Operation or the R-ii-Operation depending on which of Case i or Case ii occurs. 
Definition 4.2 (R-i-Operation to $\left.\left(T, \vec{\rho}, j, \vec{\Psi}=\vec{e}^{s-1, j}\right)\right)$. Choose the least $i \leq k$ for which either

- there exists a $\rho \supset \rho_{i l}$ such that

$$
\begin{aligned}
\left(\exists n,\left|\Psi_{i l}^{C^{(j)} \oplus \rho}(n)\right|>e_{i l}^{s-1, j} \vee[\right. & \left.\left.\Psi_{i l}^{C^{(j)} \oplus \rho}(n) \downarrow\right] \cap[Q]=\emptyset\right) \\
& \wedge(\operatorname{set}(\rho) \subseteq A) \wedge\left(\exists X \in[T], \operatorname{set}(\rho) \subseteq \pi_{i}^{k}(X) / \rho_{i l}\right)
\end{aligned}
$$

or

- there exists a $\rho \supset \rho_{\text {ir }}$ satisfying a similar condition as above but with $\operatorname{set}(\rho) \subseteq A$ replaced by $\operatorname{set}(\rho) \subseteq \bar{A}$ and "il" replaced by "ir".

If such a $\rho$ exists we say Case i occurs to $R(\cdots ; j)$. For each subcase we say that the left-hand side of part $i$ steps forward and that $R(\cdots ; j)$ steps forward on the left-hand side; or that the right-hand side of part $i$ steps forward and that $R(\cdots ; j)$ steps forward on the right-hand side. We say that $R(\cdots ; j)$, part $i$ steps forward no matter which side steps forward.

Then update initial segment requirements (according to $\rho, i)$, i.e. set $\rho_{i l}\left(\rho_{i r}\right)=\rho$ in the first (second) subcase, and update progress information:

$$
\begin{aligned}
& e_{i l}^{s-1, m}=e_{i l}^{s-1, m} \text { and } e_{i r}^{s-1, m}=e_{i r}^{s-1, m} \text { if } m \neq j, \\
& e_{i r}^{s-1, j}=e_{i r}^{s-1, j}+1 \text { and } e_{i l}^{s-1, j}=e_{i l}^{s-1, j} \text { if the right-hand side steps forward, } \\
& e_{i l}^{s-1, j}=e_{i l}^{s-1, j}+1 \text { and } e_{i r}^{s-1, j}=e_{i r}^{s-1, j} \text { if the left-hand side steps forward }
\end{aligned}
$$

and $\vec{\Psi}=\vec{e}^{s-1, j}$ in any case.

Definition 4.3 (R-ii-Operation to $\left.\left(T, \vec{\rho}, j, \vec{\Psi}=\vec{e}^{s-1, j}\right)\right)$. For a clopen set $W$, let

$$
\begin{aligned}
{\left[T_{W}\right]=\left\{X=\bigoplus_{i=1}^{k}\left(X_{i l}\right.\right.} & \left.\oplus X_{i r}\right): \bigoplus_{i=1}^{k}\left(X_{i l} \cup X_{i r}\right) \in[T] \\
& \left.\wedge \vec{\Psi}^{C^{(j)} \oplus \vec{\rho}} \text { doesn't abandon } W \text { on } \bigoplus_{i=1}^{k}\left(X_{i l} \oplus X_{i r}\right)\right\} \neq \emptyset .
\end{aligned}
$$

In this operation we find a $k^{\prime}$-disperse sequence of clopen sets $V^{(1)}, V^{(2)}, \ldots, V^{(n)}$, where $k^{\prime}=\sum_{i=1}^{k} e_{i l}^{s-1, j}+e_{i r}^{s-1, j}$ such that for all $m \leq n,\left[T_{V^{(m)}}\right] \neq \emptyset$.

If such a $k^{\prime}$-disperse sequence can be found, we say Case ii occurs. We will show in Lemma 4.6 that if Case i fails to occur, then Case ii must occur, as otherwise we obtain a $C^{(j)}$-computable constant-bound-enumeration of $Q$.

Define $\overrightarrow{\mathcal{K}}$ : for $i=1,2, \ldots, 2 k$,

(1) if $i$ is even, then let $i^{\prime}=\frac{i}{2}$ and define

$$
\mathcal{K}_{i}=\left\{K \subseteq\{1,2, \ldots, n\}:\left\{V^{(m)}\right\}_{m \in K} \text { is an } e_{i^{\prime}, l}^{s-1, j} \text {-disperse class }\right\} ;
$$

(2) if $i$ is odd, then let $i^{\prime}=\frac{i+1}{2}$ and define

$$
\mathcal{K}_{i}=\left\{K \subseteq\{1,2, \ldots, n\}:\left\{V^{(m)}\right\}_{m \in K} \text { is an } e_{i^{\prime}, r}^{s-1, j} \text {-disperse class }\right\} ;
$$

and let $u=\sum_{i=1}^{2 k}\left|\mathcal{K}_{i}\right|$.

Update $T: T=T_{s}=\operatorname{Cross}\left(T_{V^{(1)}}, T_{V^{(2)}}, \ldots, T_{\left.V^{(n)}\right)} ; \overrightarrow{\mathcal{K}}\right) ;$ update $k=k_{s}=u$. (Note that each new part is contained in an old part of some path through $T$. 
Call a new part $i^{\prime}$ a left child of an old part $i$ iff for any $X \in\left[T_{s}\right], \pi_{i^{\prime}}^{k_{s}}(X)$ is the intersection of $\pi_{i l}^{k_{s-1}}\left(X^{(p)}\right), p \in K_{2 i, m}$ for some $K_{2 i, m}$, where $X^{(p)} \in\left[T_{V^{(p)}}\right]$; and similarly for the right-hand side with $2 i$ replaced by $2 i-1$.)

Update progress information: for $i=1,2, \ldots, u$, let

$e_{i l}^{s, m}=e_{i l}^{s-1, m}, e_{i r}^{s-1, m}=e_{i r}^{s-1, m}$ if $m \neq j$,

$e_{i r}^{s, j}=e_{i^{\prime} r}^{s-1, j}+1, e_{i l}^{s-1, j}=e_{i^{\prime} l}^{s-1, j}$ if part $i$ of $T_{s}$ is a right child of part $i^{\prime}$ of $T_{s-1}$,

$e_{i l}^{s, j}=e_{i^{\prime} l}^{s-1, j}+1, e_{i r}^{s-1, j}=e_{i^{\prime} r}^{s-1, j}$ if part $i$ of $T_{s}$ is a left child of part $i^{\prime}$ of $T_{s-1}$.

Finally, assign the value $\vec{e}^{s, t_{s+1}}$ to $\vec{\Psi}$.

4.2. Construction. Let $C^{(j)}$ be a sequence of sets of $\mathcal{M}$ that is cofinal in $\mathcal{M}$, i.e. $(\forall j \in \mathbb{N})\left[C^{(j)} \in \mathcal{M} \wedge\left(\forall C \in \mathcal{M} \exists C^{(j)}, C \leq_{T} C^{(j)}\right)\right]$; furthermore, we require that $\forall j \in \mathbb{N}, C^{(j)} \leq_{T} C^{(j+1)}$. Fix a sequence of elements of $\left\{C^{(j)}\right\}_{j \in \mathbb{N}}$, namely $C^{\left(t_{s}\right)}$, such that each $C^{(j)}$ appears infinitely often in this sequence, i.e. $\exists^{\infty} s, t_{s}=j$.

Now suppose we are at the beginning of Step $s$, we have a $C$-computable $k_{s-1^{-}}$ partition tree $T=T_{s-1}$ with $[T] \neq \emptyset$, and $C^{(j)}$ is the corresponding set in the $s^{\text {th }}$ position of the sequence (i.e. $t_{s}=j$, so Step $s$ is devoted to $R(\cdots ; j)$ ), with current progress $\vec{\Psi}=\vec{e}^{s-1, j}$ for $R(\cdots ; j)$ and initial segment requirements $\vec{\rho}$.

Begin Step $s$ of the construction:

(1) Repeatedly apply the R-i-Operation to $\left(T, \vec{\rho}, j, \vec{\Psi}=\vec{e}^{s-1, j}\right)$ until Case i fails to occur to $R(\cdots ; j)$.

(We will show in Lemma 4.4 that after finitely many repetitions Case i will stop occurring).

(2) Then apply the R-ii-Operation to $\left(T, \vec{\rho}, j, \vec{\Psi}=\vec{e}^{s-1, j}\right)$.

(We will show in Lemma 4.6 that if Case i doesn't occur, then Case ii must occur, as otherwise we obtain a strong constant-bound-enumeration of $Q$.)

(3) For each part $i, i \leq k_{s}$, if part $i$ is a left child, then apply the P-Operation to the left-hand side of $T_{s}$ 's part $i$; otherwise apply the P-Operation to the right-hand side of $T_{s}$ 's part $i$.

(Here the P-Operation is applied to each part in order of their indices. We will show in Lemma 4.5 that the P-Operation succeeds on at least one of the new parts. Note that on each "new" part at least one side of $R(\cdots ; j)$ has just stepped forward.)

(4) Finally, go to the next step.

Note that in either case $R(\cdots ; j)$ only steps forward but not "backward", since each child part is a subset of its parent part, and the initial segment requirements are inherited.

4.3. Verification. We need the following three facts as mentioned above.

Lemma 4.4. Within each step, Case $i$ will not occur to $R(\cdots ; j)$ forever.

Lemma 4.5. For any $A$ and any $C$-computable ordered $k$-partition tree $T$ such that $[T] \neq \emptyset$, if $A \varliminf_{T} C$, then there exist $i \leq k$ and $X \in[T]$ such that both $\pi_{i}^{k}(X) \cap A$ and $\pi_{i}^{k}(X) \cap \bar{A}$ are non-empty, which implies that in Case ii, for at least one of the new parts, the P-Operation succeeds on the side of that part to which it is applied. 
Lemma 4.6. Let $E$ be a set of finite subsets of $2^{<\omega}$ c.e. in e such that, for all $n$ :

(1) $\mathcal{W}_{n}=\left\{W \subseteq 2^{<\omega}: \forall \rho \in W,|\rho|=n \wedge W \notin E\right\}$ is not a $k^{\prime}$-disperse class.

(2) $Q_{n}=\left\{\rho \in 2^{<\omega}:|\rho|=n \wedge[\rho] \cap[Q] \neq \emptyset\right\} \notin E$.

Then there exists a strong $k^{\prime}$-enumeration $h: \omega \rightarrow \omega$ of $Q$ computable in $\mathbf{e}$.

Actually, we could further require that $\left(\forall \rho \in D_{h(n)}\right)|\rho|=n$. This implies that for any specific tuple $\left(T, \vec{\rho}, j, \vec{\Psi}=\vec{e}^{s-1, j}\right)$ in the construction, either Case $i$ or Case ii occurs since otherwise $E=\left\{W \subseteq 2^{<\omega}: \forall \rho, \sigma \in W,|\rho|=|\sigma|,\left[T_{W}\right]=\emptyset\right\}$ is a $C \oplus C^{(j)}$-c.e. set which satisfies (1) and (2) (recall item (2) in the Note to Step $s$ and the definition of $R$-ii-Operation).

Assuming these facts we now show that the construction does provide the desired $G$. Note that our construction yields a tree $\mathcal{T}$ : Each node at the $s^{\text {th }}$ level represents a part at the $s^{\text {th }}$ step and corresponds to a tuple $\left(j ; e_{l}, e_{r}\right)$ standing for the progress of Step $s$ for $C^{(j)}$ (on this part), where $C^{(j)}$ is the set that this step is devoted to, i.e. the $j$ such that $R(\cdots ; j)$ steps forward at this step. The successor nodes of a node $c$ are those representing parts that are descendant parts of the part that $c$ represents. Clearly $\mathcal{T}$ is a finitely branching tree.

Definition 4.7. Say part $i$ fades away on the left-hand side at Step $s$ iff $\forall X \in$ $\left[T_{s}\right], \pi_{i}^{k}(X) \cap A=\emptyset$ (and similarly for the right-hand side with $\bar{A}$ replacing $A$ ).

Note that if part $i$ 's left-hand side fades away at Step $s$, then it fades away forever; i.e. all its descendants also fade away at the left-hand side. So by Lemma 4.5 there exists an infinite subtree $\mathcal{T}^{\prime}$ of $\mathcal{T}$ such that each $s^{\text {th }}$ level node of $\mathcal{T}^{\prime}$ represents a part that has not yet faded away on either side at Step $s$. Therefore there exists a path $f$ through this subtree. Note that for each $R(\cdots ; j)$ there exists some side, say the right-hand side, such that along $f, R(\cdots ; j)$ steps forward on this side infinitely often. Therefore there exists some side, say the right-hand side, such that for any $C^{(j)}$ there exists $C^{\left(j^{\prime}\right)}$ with $C^{(j)} \leq_{T} C^{\left(j^{\prime}\right)}$, and there exist infinitely many steps $s_{m}, m \in \mathbb{N}$, such that step $s_{m}$ is devoted to $R\left(\cdots ; j^{\prime}\right), f\left(s_{m}\right)$ 's represented part $f_{s_{m}}$ steps forward at the right-hand side at step $s_{m}$, and the following P-Operation (applied to the new part corresponding to this part's right-hand side) succeeds. In other words, for any $C \in \mathcal{M}$ there exists $C^{\left(j^{\prime}\right)}$ such that $C \leq_{T} C^{\left(j^{\prime}\right)}$ and along $f$, $R\left(\cdots ; j^{\prime}\right)$ steps forward on the right-hand side infinitely often (which implies that all $R\left(j ; e_{r}\right), e_{r} \in \mathbb{N}$ are satisfied) and the P-Operation succeeds (on the right-hand side) infinitely often. Therefore, $G=\bigcup_{s=1}^{\infty} \rho_{f_{s}, r}^{s}$ is infinite and $C^{\left(j^{\prime}\right)} \oplus G$ doesn't compute a constant-bound-enumeration of $Q$, so that $G \oplus C \leq_{T} G \oplus C^{\left(j^{\prime}\right)}$ also fails to compute a strong constant-bound-enumeration of $Q$, i.e. $\forall C \in \mathcal{M}, G \oplus C$ doesn't compute a strong constant-bound-enumeration of $Q$.

It remains to prove the three facts mentioned above.

Proof of Lemma 4.4. Recall that there are infinitely many Turing functionals $\Psi_{e}$ such that, for any oracle $C \oplus G, \Psi_{e}^{C \oplus G}$ doesn't halt on any input. Call such Turing functionals trivial. Clearly, at Step $s$, if a part proceeds to $\left(e_{l}, e_{r}\right)$ (during the Case i loop), where $\Psi_{e_{l}}$ is a trivial Turing functional, then the R-i-Operation will never succeed on the left-hand side of this part, and similarly for the right-hand side if $\Psi_{e_{r}}$ is trivial. Since there are only finitely many different parts and sides, and the R-i-Operation doesn't increase the total number of parts, either Case i fails to occur during the loop or all Turing functionals in the progress tuples will finally 
be updated to trivial ones, which also causes Case i to fail to occur, since Case i occurring implies that at least some Turing functional halts on some input.

Proof of Lemma 4.5. Suppose on the contrary that $(\forall i \leq k)\left(\left[\forall X \in[T], \pi_{i}^{k}(X) \cap\right.\right.$ $\left.A=\emptyset] \vee\left[\forall X \in[T], \pi_{i}^{k}(X) \cap \bar{A}=\emptyset\right]\right)$. Let $R=\left\{i \leq k: \forall X \in[T], \pi_{i}^{k}(X) \cap A=\emptyset\right\}$ and $L$ be the set of all $i \leq k$ that are not in $R$. We show that $A$ is computable in $C$. Note that $T$ is a partition of $W={ }^{*} \omega=\omega-F$ for some finite set $F$, i.e. $\forall X \in[T], \bigcup_{i=1}^{k} \pi_{i}^{k}(X) \supseteq \omega-F$. To decide whether $n \in A$, suppose $n \notin F$, wait (using $C$ ) for a moment such that for some $m, \forall \rho,(|\rho|=m \wedge \rho \in T) \Rightarrow[(\forall i \in L, n \notin$ $\left.\left.\pi_{i}^{k}(\rho)\right) \vee\left(\forall i \in R, n \notin \pi_{i}^{k}(\rho)\right)\right] \vee[\exists N \in \mathbb{N}, \forall \sigma \supset \rho,|\sigma|=N \rightarrow \sigma \notin T]$. Note that such a moment must exist by the assumption that each part is contained in either $A$ or $\bar{A}$, so $n \in A \rightarrow\left[\left(\forall i \in R, n \notin \pi_{i}^{k}(\rho)\right) \vee(\exists N \in \mathbb{N}, \forall \sigma \supset \rho,|\sigma|=N \rightarrow \sigma \notin T)\right]$ and similarly for $n \in \bar{A}$. Furthermore, since $\forall X \in[T], \bigcup_{i=1}^{k} \pi_{i}^{k}(X) \supseteq \omega-F$, we have $\left(\forall i \in L, n \notin \pi_{i}^{k}(\rho)\right) \rightarrow n \in \pi_{j}^{k}(\rho)$ for some $j \in R$ and $\rho \subset X \in[T]$. Therefore $n \in A$ iff $\exists m \forall \rho,(|\rho|=m \wedge \rho \in T) \Rightarrow\left(\forall i \in R, n \notin \pi_{i}^{k}(\rho)\right)$ and $n \in \bar{A}$ iff $\exists m \forall \rho,(|\rho|=m \wedge \rho \in T) \Rightarrow\left(\forall i \in L, n \notin \pi_{i}^{k}(\rho)\right)$. So we could compute $A-F$ using $C$, which is clearly equivalent to $A \leq_{T} C$.

Proof of Lemma 4.6. We first show that if neither Case i nor Case ii occurs, then $E=\left\{W \subseteq 2^{<\omega}: \forall \rho, \sigma \in W,|\rho|=|\sigma|,\left[T_{W}\right]=\emptyset\right\}$ is $C \oplus C^{(j)}$-c.e. and satisfies (1) and (2). Note that $E$ is clearly $C \oplus C^{(j)}$-c.e.

Clearly, if Case ii does not occur, then $E$ satisfies (1) by the definition of "Case ii occurs". Suppose for some $Q_{n},\left[T_{Q_{n}}\right]=\emptyset$. Consider an arbitrary $X \in[T]$. For $i \leq k$ let $X_{i l}=\pi_{i}^{k}(X) \cap A$ and $X_{i r}=\pi_{i}^{k}(X) \cap \bar{A}$. Since $\left[T_{Q_{n}}\right]=\emptyset, X^{\prime}=\bigoplus_{i \leq k}\left(X_{i l} \oplus X_{i r}\right) \notin$ $\left[T_{Q_{n}}\right]$. Therefore on some part $i, \vec{\Psi}_{i}{ }^{C^{(j) \oplus \overrightarrow{\rho_{i}}}}$ abandons $Q_{n}$ on $X_{i l} \oplus X_{i r}$. Thus, by the definition of abandoning, item (2), either $\left(\exists \rho \supset \rho_{i l}\right.$, set $(\rho) \subseteq X_{i l} \subseteq A, \exists n \in$ $\mathbb{N})\left[\Psi_{i}^{C^{(j)} \oplus \rho}(n) \downarrow\right] \cap\left[Q_{n}\right]=\emptyset \vee\left|\Psi_{i l}^{C^{(j)} \oplus \rho}(n)\right|>$ index of $\left.\Psi_{i l}\right)$ or $\left(\exists \rho \supset \rho_{i r}, \operatorname{set}(\rho) \subseteq\right.$ $\left.X_{i r} \subseteq \bar{A}, \exists n \in \mathbb{N}\right)\left[\Psi_{i r}^{C^{(j)} \oplus \rho}(n) \downarrow\right] \cap\left[Q_{n}\right]=\emptyset \vee\left|\Psi_{i l}^{C^{(j)} \oplus \rho}(n)\right|>$ (index of $\Psi_{i l}$ ). However, this implies that Case i occurs.

Now we show that the algorithm of $E$ (or equally speaking, the degree e) can be used to compute a strong $k$-enumeration of $Q$. Let $E_{t}$ denote $E$ at stage $t$ and $\mathcal{W}_{n, t}=\left\{W \subseteq 2^{<\omega}: \forall \rho \in W,|\rho|=n \wedge W \notin E_{t}\right\}$. Note that since $E$ is an enumeration, $\mathcal{W}_{n, t+1} \subseteq \mathcal{W}_{n, t}$. Therefore, if there exists a $k^{\prime}$-partition of $\mathcal{W}_{n}$, $P^{(1)} \cup P^{(2)} \cup \cdots \cup P^{\left(k^{\prime}\right)}=\mathcal{W}_{n}$, such that $(\forall i)\left(\bigcap_{W \in P^{(i)}} W \neq \emptyset\right)$, then this partition can be found in a finite amount of time. Furthermore, $Q_{n} \in P^{(i)}$ for some $i$, so $\forall \rho \in \bigcap_{W \in P^{(i)}} W, \rho \in Q$. It follows that the function $h$ defined below, which is computable using an algorithm for $E$ (i.e. computable in any degree that computes $E)$, is a strong $k^{\prime}$-enumeration of $Q$ :

$$
\begin{aligned}
D_{h(n)}= & \left\{\rho_{1}, \rho_{2}, \ldots, \rho_{k^{\prime}}: \text { there exists some } t\right. \\
& \text { and a } k^{\prime} \text {-partition of } \mathcal{W}_{n, t}=\bigcup_{i=1}^{k^{\prime}} P^{(i)} \text { s.t. }(\forall j)\left(\bigcap_{W \in P^{(j)}} W \neq \emptyset\right) ;
\end{aligned}
$$

for $i=1,2, \ldots, k^{\prime}, \rho_{i}$ is the leftmost string in $\left.\bigcap_{W \in P^{(i)}} W\right\}$. 


\section{Applications}

\section{Corollary 5.1. Over $\mathrm{RCA}_{0}, \mathrm{RT}_{2}^{2}$ does not imply $\mathrm{WWKL}_{0}$.}

Before proving this corollary, we make some remarks. It is known that in $\omega$ models $\mathrm{WWKL}_{0}$ is equivalent to the assertion that for each $X$ there is a set that is Martin-Löf random relative to $X$. It is known that $X \in 2^{\omega}$ is Martin-Löf random iff $(\forall n) K\left(X\lceil n) \geq^{+} n\right.$, where $K(\rho)$ denotes the prefix-free Kolmogorov complexity of a string, and furthermore there exists a universal prefix-free machine $U$ such that for every prefix-free machine $M, K_{U} \leq K_{M}+c$ for some constant depending on $M$. For more background on algorithmic randomness theory, see [5, 19]. Fix a universal prefix-free machine $U$ in the following text.

Definition 5.2. A string $\rho$ is $c$-incompressible $U$ iff $K_{U}(\rho) \geq|\rho|-c$.

Note that $\left[T_{c}\right]=\left\{X \in 2^{\omega}: \forall n, X\left\lceil n\right.\right.$ is c-incompressible $\left.{ }_{U}\right\}$ is a closed set of $2^{\omega}$.

Lemma 5.3. For any positive integers $k, c$, there is no computable strong $k$-enumeration of the $c$-incompressible strings. $_{\text {- }}$.

Proof. Suppose on the contrary that $h: \omega \rightarrow \omega$ is a computable strong $k$-enumeration of the $c$-incompressible $U$ strings. Let $\sigma_{n}=\underbrace{00 \ldots 0}_{n} 1$. We define a prefix-free machine $M$. The algorithm of $M$ is as follows for each $n$ : look for an $m \geq n^{2}$, and then output $\rho_{i}$ on input $\sigma_{k n+i}$ (note that $j \leq k$ ). Note that all strings $\rho_{1}, \ldots, \rho_{j} \in$ $D_{h(m)}$ have length $m=n^{2}$. Since $U$ is universal, there exists a constant $d$ such that $K_{U}\left(\rho_{i}\right)<k n+i+d$; therefore, for sufficiently large $n, \forall i \leq k, K_{U}\left(\rho_{i}\right)<$ $\left|\rho_{i}\right|-c$, contradicting the assumption that $h$ is a strong $k$-enumeration of the $c$ incompressible $_{U}$ strings. $^{2}$

Note that although Theorem 1.2 proves the cone avoidance result for one closed set $[Q]$, it can be easily adapted to construct $G$ cone avoiding countably many closed sets as long as for every finite number of them, $Q_{1}, Q_{2}, \ldots, Q_{n}$, the joint union of them, $\left[\bigvee_{i=1}^{n} Q_{i}\right]=\left\{X \in 2^{\omega}:\left(\exists j \leq n \exists Y \in\left[Q_{j}\right]\right) X=\rho_{j} * Y\right\}$, where $\rho_{j}$ is determined by $Q_{j}$, satisfies the condition in Theorem 1.2

Now we can prove the main theorem.

Proof of Corollary [5.1. It suffices to construct a countable class $\mathcal{M}$ satisfying four conditions: (a) $C, B \in \mathcal{M} \rightarrow C \oplus B \in \mathcal{M}$; (b) $\left(C \in \mathcal{M} \wedge B \leq_{T} C\right) \rightarrow B \in \mathcal{M}$; (c) $(\forall C \in \mathcal{M}) C$ does not compute a strong constant-bound-enumeration of any $T_{c}$; (d) $\mathcal{M} \vDash \mathrm{RT}_{2}^{2}$.

Note that (a) and (b) ensure that $R C A_{0}$ is satisfied, (c) ensures that $W W K L_{0}$ is not satisfied, and (d) ensures that $\mathrm{RT}_{2}^{2}$ is satisfied.

It is shown in [4, Lemma 7.11] and later corrected in [3] that $\mathrm{RCA}_{0}+\mathrm{RT}_{2}^{2}$ is equivalent to $\mathrm{RCA}_{0}+\mathrm{SRT}_{2}^{2}+\mathrm{COH}$. Here $\mathrm{COH}$ is the axiom saying that for any $C=\bigoplus_{i=0}^{\infty} C_{i} \in \mathcal{M}$ there exists an infinite set $C^{*}$ cohesive for $C$; i.e. for any component $C_{i}$ either $C^{*}-C_{i}$ or $C^{*}-\bar{C}_{i}$ is finite.

Moreover, we now show that it can be proved by the finite extension method that if $\left\{R^{(i)}\right\}_{i \in \mathbb{N}}$ is a sequence such that no finite disjoint union of the $R^{(i)}$ computes a strong constant-bound-enumeration of any $T_{c}$, then there exists an infinite set $G$ cohesive for $\left\{R^{(i)}\right\}_{i \in \mathbb{N}}$ that also does not compute any strong constant-boundenumeration of any $T_{c}$. To construct such a cohesive set, we try to satisfy the 
following requirements.

$$
\begin{aligned}
& R_{e}: \Psi_{e}^{G} \text { is not a strong } e \text {-enumeration of any } T_{c}, \\
& N_{i}: G \subseteq^{*} R^{(i)} \vee G \subseteq^{*} \omega-R^{(i)}
\end{aligned}
$$

Clearly we can inductively define a $0-1$ sequence $\nu$ such that, letting $R^{i}=R^{(i)}$ if $\nu(i)=1$ and $R^{i}=\omega-R^{(i)}$ if $\nu(i)=0$, we have $(\forall n)\left|\bigcap_{i=0}^{n} R^{i}\right|=\infty$.

For each Step $s$, let $G_{s}=\bigcap_{i=0}^{n} R^{i}$; we construct an initial segment $\rho_{s}$ such that $\rho_{s} \supset \rho_{s-1}, \operatorname{set}\left(\rho_{s}\right) \subseteq \operatorname{set}\left(G_{s-1} / \rho_{s-1}\right)$, and furthermore, $\left(\rho_{s}, G_{s}\right)$, as a Mathias forcing condition, forces $R_{s}$ and $N_{s}$. It is clear that for any $\rho,\left(\rho, G_{s}\right)$ forces $N_{s}$. We now choose $\rho \supset \rho_{s-1} \wedge \operatorname{set}(\rho) \subseteq G_{s-1}$ to satisfy $R_{s}$. There are two ways to do so: find a $\rho$ with $\rho \supset \rho_{s-1} \wedge \operatorname{set}(\rho) \subseteq G_{s-1}$ such that $\Psi_{e}^{\rho}(n)$ does not halt, or one such that $\left|\Psi_{e}^{\rho}(n) \downarrow\right|>e \vee \Psi_{e}^{\rho}(n) \cap T_{c}=\emptyset \vee\left(\left(\exists \sigma \in \Psi_{e}^{\rho}(n)\right)|\sigma|>n\right)$. Suppose we can't find such $\rho$; this implies that for every $n$, there exists $\rho$ with $\rho \supset \rho_{s-1} \wedge \operatorname{set}(\rho) \subseteq G_{s-1}$ such that $\left|\Psi_{e}^{\rho}(n) \downarrow\right| \leq e$ and $\Psi_{e}^{\rho}(n)$ is a set of length $n$ strings that has nonempty intersection with $T_{c}$. But then one could use this $\Psi_{e}$ and oracle $\bigoplus_{i=0}^{s} R^{(i)}$ to compute a strong $e$-enumeration of $T_{c}$, a contradiction.

In the following text, for a class of sets $\mathcal{M}$, we say $X$ is $\mathcal{M}$-computable iff $\exists C \in \mathcal{M}$ s.t. $X \leq_{T} C$. The notions of $\mathcal{M}$-effectively closed, $\Pi_{1}^{0, \mathcal{M}}$, etc. are defined similarly. Note that the conclusion just proved above is relativizable to any countable class of sets closed under join. Let $\mathcal{M}_{0}=$ all recursive sets. Let $f$ be a computable stable coloring. By Theorem 1.2, there exists an infinite $G_{0}$ with $G_{0} \subseteq f_{1} \vee G_{0} \subseteq f_{2}$ such that $\forall C \in \mathcal{M}_{0}$, for each $c, C \oplus G_{0}$ does not compute any strong constant-boundenumeration of any infinite subset of any $T_{c}$. Let $\mathcal{M}_{1}=\left\{X \in 2^{\omega}: X \leq_{T} C \oplus G_{0}\right.$ for some $\left.C \in \mathcal{M}_{0}\right\}$. Clearly $\mathcal{M}_{1}$ satisfies (a)(b)(c). Let $G_{1}$ be cohesive for a sequence of uniformly $\mathcal{M}_{1}$-computable sets (where $\mathcal{M}_{1}$-computable means computable in some $\left.C \in \mathcal{M}_{1}\right)$, so that $\left(\forall C \in \mathcal{M}_{1}\right) G_{1} \oplus C$ does not compute any strong constantbound-enumeration of any infinite subset of any $T_{c}$. Let $\mathcal{M}_{2}=\left\{X \in 2^{\omega}: \exists C \in\right.$ $\left.\mathcal{M}_{1}, X \leq_{T} C \oplus G_{1}\right\}$. Clearly $\mathcal{M}_{2}$ also satisfies (a)(b)(c). Iterate the above process to ensure that (1) for any uniformly $\mathcal{M}_{j}$-computable sequence $C^{(1)}, C^{(2)}, \ldots$, there exists an $i$ and a $G_{i-1} \in \mathcal{M}_{i}$ such that $G_{i-1}$ is cohesive for $C^{(1)}, C^{(2)}, \ldots$ and (2) for any $C \in \Delta_{2}^{0, \mathcal{M}_{j}}\left(\Delta_{2}^{0, \mathcal{M}_{j}}\right.$ means $\Delta_{2}^{0, C}$ for some $\left.C \in \mathcal{M}_{j}\right)$, there exists an $i$ and an infinite $G_{i-1} \in \mathcal{M}_{i}$ such that $G_{i-1} \subseteq C \vee G_{i-1} \subseteq \bar{C}$. It follows that $\mathcal{M}=\bigcup_{i=0}^{\infty} \mathcal{M}_{i} \vDash \mathrm{RCA}_{0}+\mathrm{SRT}_{2}^{2}+\mathrm{COH} \Leftrightarrow \mathrm{RCA}_{0}+\mathrm{RT}_{2}^{2}$, but clearly $\mathcal{M}$ satisfies (a) (b)(c). The conclusion follows.

We now prove Corollary 1.11

Corollary 5.4. There exists a DNR function that does not compute any binary sequence with positive effective Hausdorff dimension.

Proof. Again note that the construction can be adapted to construct a set $G$ cone avoiding countably many closed sets if every finite joint union of those sets satisfies the conditions of Theorem [1.2. Therefore, choose a sequence $d_{n}>0$ with $\lim _{n \rightarrow \infty} d_{n}=0$ and let $A$ be a $\Delta_{2}^{0}$ set such that every infinite subset of $A$ or $\bar{A}$ computes a $D N R$ function. Now apply Theorem 1.2 to construct $G \subseteq A \vee G \subseteq \bar{A}$ that cone avoids all corresponding closed sets $\left[T_{d_{n}}\right]$, and let $f \leq_{T} G$ be a $D N R$ function. Then $f$ does not compute any binary sequence of positive effective Hausdorff dimension. 
For most "natural" trees that have been studied, if the induced closed set has Muchnik degree strictly above 0 as a mass problem, then the tree does not admit a strong constant-bound-enumeration. For example, this fact is true of homogeneous trees.

Definition 5.5. A tree $T$ is homogeneous iff $\left(\forall \rho_{1}, \rho_{2} \in T \forall \rho\right)\left(\rho / \rho_{1} \in T \Leftrightarrow \rho / \rho_{2} \in\right.$ $T)$.

Lemma 5.6. Let $T \subseteq \omega^{<\omega}$ be a finitely branching homogeneous tree, and let a be a Turing degree such that $[T]$ is a-effectively closed. Then a computes a strong $k$-enumeration of $T$ for some $k \in \mathbb{N}$ iff a computes a path through $T$.

Proof. $(\Leftarrow)$ : Straightforward, since an enumeration of the initial segments of a path through $T$ is a strong 1-enumeration of $T$.

$(\Rightarrow)$ : We prove this by induction. Let $h \leq_{T}$ a be a strong $k$-enumeration of $T$. Clearly the conclusion follows when $k=1$.

Suppose $\exists N \in \mathbb{N} \forall n>N D_{h(n)} \subset T$. Then clearly one can compute a path through $T$ as follows: Let $g(n)$ be the first integer $g_{n}$ we find such that $\exists m>$ $N \exists \sigma \in D_{h(m)}, \sigma(n)=g_{n}$. Since $T$ is homogeneous, $g$ is a path through $T$.

Suppose $\forall N \in \mathbb{N} \exists n>N D_{h(n)} \not \subset T$. Since $[T]$ is a-effectively closed, which means $\bar{T}$ is a-c.e., $\left\{n \in \mathbb{N}: D_{h(n)} \not \subset T\right\}$ is an infinite a-c.e. set. Therefore one can compute a strong $(k-1)$-enumeration of $T$ as follows: Search for an $m$ and a $\sigma$ such that $\sigma \in D_{h(m)} \cap \bar{T}_{m}$ and $\forall \rho \in D_{h(m)}|\rho| \geq n$, and let $g(n)$ be the index of $\left\{\rho \uparrow_{n}: \rho \in D_{h(m)}-\{\sigma\}\right\}$.

Note that $\left|D_{g(n)}\right| \leq k-1$ and $D_{g(n)} \cap T \neq \emptyset$ (as otherwise $\left.D_{h(m)} \cap T=\emptyset\right)$, i.e. $g \in$ $\omega^{\omega}$ is a strong $(k-1)$-enumeration of $T$. The conclusion follows by induction.

Combining Theorem 1.2 and Lemma 5.6 yields:

Corollary 5.7. Let $\mathcal{M}$ be a countable class closed under disjoint union, and let $Q \subseteq \omega^{<\omega}$ be an infinite $\mathcal{M}$-computably bounded homogeneous tree such that $[Q]$ is $\mathcal{M}$-effectively closed. Then

$$
\left(\forall A \in 2^{\omega}\right)(\exists G \text { s.t. }(G \subseteq A \vee G \subseteq \bar{A}) \wedge|G|=\infty)(\forall C \in \mathcal{M}, f \in[Q]) f \not_{T} G \oplus C
$$

if and only if $[Q] \mathbb{E}_{u} \mathcal{M}$. Here $\leq_{u}$ denotes the Muchnik reducibility.

Note that the computable boundedness is needed to ensure that in the proof of Lemma 4.6 the class $\mathcal{W}_{n}$ is $\Pi_{1}^{0, \mathcal{M}}$ uniformly in $n$, which makes sure that the enumeration algorithm is computable in $\mathcal{M}$.

For $f \in \mathbb{N}^{\mathbb{N}}, f-W_{K} L_{0}$ as a second order arithmetic statement says: for any set $X$ there exists a $g \in f-D N R^{X}$. $\mathrm{WKL}_{0}$ is $\mathbf{f}-\mathrm{WKL}_{0}$ restricted to $f \equiv 2$. Using Corollary 5.7, we have:

Corollary 5.8. For any computable $f \in \mathbb{N}^{\mathbb{N}}$ such that $f(n)>1$ i.o., $\mathrm{RT}_{2}^{2}$ does not imply $\mathrm{f}-\mathrm{WKL}_{0}$.

Proof. Exactly the same as Corollary 5.1 .

We now mention an interesting result derived by Kjos-Hanssen in algorithmic randomness theory.

Corollary 5.9 (Kjos-Hanssen [13]). For every Martin-Löf random set $A \in 2^{\omega}$, there exists an infinite set $G \subseteq A \vee G \subseteq \bar{A}$ such that $G$ does not compute any Martin-Löf random set. 
Proof. We've already shown in Lemma 5.3 that every finite joint union of the countably many effectively closed sets $\left[T_{c}\right]=\left\{X \in 2^{\omega}: \forall n, X\left\lceil n\right.\right.$ is c-incompressible $\left._{U}\right\}$ satisfies the condition of Theorem 1.2. So the conclusion follows from Theorem 1.2 directly.

\section{REMARKS}

Remark 6.1. Although at each step of the construction we hold an $\mathcal{M}$-computable tree $T$, updated by the R-ii-operation and the P-operation, this is not essential. One could pre-choose a path through $T$. Nonetheless, the construction tree $\mathcal{T}$ is not avoidable. Furthermore, holding the tree requires less computational power of the constructor.

Remark 6.2. Can we cone avoid an arbitrary tree $Q$ if $Q$ is not Muchnik reducible to $\mathcal{M}$ ? We guess not, and a construction showing that the answer is no might be provided by modifying the proof of Theorem 2.3 of [10], and noting that Part reduces a $k$-enumeration to a 1 -enumeration.

Remark 6.3. We have modularized the construction into as many parts as we could, which makes it convenient for further applications and generalizations. For example, by modifying the R-ii-Operation and analyzing the proof of Lemma 4.5. one might strengthen Corollary 5.9 to prove Kjos-Hanssen's result in 14 that any Martin-Löf random set contains an infinite subset that does not compute any Martin-Löf random set.

Another kind of application is to let Part $t_{k}$ serve as a mediate as in Corollaries 1.9 and 1.11, i.e. first one proves that some class $\mathbb{X}$ is Muchnik reducible to Part $_{k}$, and then it follows that some member of $\mathbb{X}$ does not compute any member of another class.

Remark 6.4. A not so trivial generalization is to reinterpret $\subseteq, \cap$ and therefore overload Cross and the definition of "abandon". This is explained as follows.

Say that we can strongly compute a class $\mathcal{Q}$ under the condition $\langle\mathcal{B}, \mathcal{C}(A)\rangle$, where $\mathcal{B}, \mathcal{C}(A)$ are classes, possibly with other "parameters", iff $\exists A \in \mathcal{B} \quad \mathcal{Q} \leq_{u} \mathcal{C}(A)$. (In this paper $\mathcal{B}=\mathcal{P}(\omega), \mathcal{C}(A)=\operatorname{Part}_{2}(A), \mathcal{Q}$ is a closed set with $Q$ non- $k$-enumerable in $\mathcal{M}$, and we could not strongly compute $\mathcal{Q}$ under the condition $\langle\mathcal{B}, \mathcal{C}(A)\rangle$ for any $A$.) Therefore $G \subseteq A$ is generalized as $G \in \mathcal{C}(A)$ and $X \cap Y$ is generalized as $\mathcal{C}(X) \cap \mathcal{C}(Y)$. Note also that in many applications, $\mathcal{B}, \mathcal{C}(A)$ are $\Pi_{1}^{0}$ classes, for example $\mathcal{B}=\left\{T \subseteq 2^{<\omega}: T\right.$ is a tree satisfying certain property $\}$, say the property of being $n$-bushy, and $\mathcal{C}(T)=[T]$ (or almost $\Pi_{1}^{0}$ as $\operatorname{Part}_{2}(A)$, which is a $\Pi_{1}^{0}$ class minus a countable class of sets). Therefore by redefining "abandon" and Cross, the main frame of the construction may still serve for this purpose. (Of course, different combinatorial facts will be needed in different cases and there would be no universal way to produce these combinatorial facts; that belongs to the creative part of mathematics. However, there might be some results with broad application, since what we have studied is only a stone beside the sea.) We hope that this frame could help in the study of homogeneous trees, especially $f$-DNR, and give universal solutions to various results that have arisen recently, for example in [9, 18; see also [15].

Question 6.5. The most important question at hand is what kind of $\Pi_{3}^{0}$ class can be avoided as above. Note that this is related to the question of whether $\mathrm{SRT}_{2}^{2}$ 
implies $\mathrm{RT}_{2}^{2}$, which is equivalent to whether $\mathrm{SRT}_{2}^{2}$ implies $\mathrm{COH}$. Here $\mathrm{COH}$ is the second order arithmetic statement that every sequence of sets $R^{(i)}$ has a cohesive set for it, i.e. an infinite set $Y$ such that for all $i \in \mathbb{N}$ either $Y \cap R^{(i)}$ or $Y \cap \bar{R}^{(i)}$ is finite. Note that the class of cohesive sets of a given $R^{(i)}$ is a $\Pi_{3}^{0, R^{(i)}}$ class.

Question 6.6. What is the difference between the Muchnik lattice of Part 2 and that of Part $_{3}$, Part $_{k}$ ? (Here the Muchnik lattice of a collection of subsets of $\omega^{\omega}$ refers to the lattice where the $\leq$ relation is induced by the Muchnik reducibility.)

For example, is there a $\operatorname{Part}_{3}\left(A_{1}, A_{2}\right)$ such that there exists no $\operatorname{Part}_{2}(A)$ to which $\operatorname{Part}_{3}\left(A_{1}, A_{2}\right)$ is Muchnik reducible? For the latter question we guess the answer is yes.

\section{ACKNOWLEDGEMENT}

The author is grateful to Professor Peter Cholak for his help during the preparation of this paper.

\section{REFERENCES}

[1] Klaus Ambos-Spies, Bjørn Kjos-Hanssen, Steffen Lempp, and Theodore A. Slaman, Comparing DNR and WWKL, J. Symbolic Logic 69 (2004), no. 4, 1089-1104, DOI 10.2178/jsl/1102022212. MR2135656 (2006c:03061)

[2] Richard Beigel, Harry Buhrman, Peter Fejer, Lance Fortnow, Piotr Grabowski, Luc Longpre, Andrej Muchnik, Frank Stephan, and Leen Torenvliet, Enumerations of the Kolmogorov function, J. Symbolic Logic 71 (2006), no. 2, 501-528, DOI 10.2178/jsl/1146620156. MR2225891 (2007b:68086)

[3] P.A. Cholak, C.G. Jockusch, and T.A. Slaman, Corrigendum to: On the strength of Ramsey's theorem for pairs, J. Symbolic Logic 74 (2009), no. 4, 1438-1439. MR2583829

[4] On the strength of Ramsey's theorem for pairs, Journal of Symbolic Logic 66 (2001), no. 1, 1-55. MR.1825173

[5] Rodney G. Downey and Denis R. Hirschfeldt, Algorithmic randomness and complexity, Theory and Applications of Computability, Springer, New York, 2010. MR2732288(2012g:03001)

[6] Damir D. Dzhafarov and Carl G. Jockusch Jr., Ramsey's theorem and cone avoidance, J. Symbolic Logic 74 (2009), no. 2, 557-578, DOI 10.2178/jsl/1243948327. MR2518811 (2010e:03052)

[7] Harvey Friedman, Some systems of second order arithmetic and their use, Proceedings of the International Congress of Mathematicians (Vancouver, B. C., 1974), Vol. 1, Canad. Math. Congress, Montreal, Que., 1975, pp. 235-242. MR.0429508 (55 \#2521)

[8] Harvey Friedman, Systems of second order arithmetic with restricted induction, $i$, ii, Journal of Symbolic Logic 41 (1976), no. 2, 557-559.

[9] Noam Greenberg and Joseph S. Miller, Diagonally non-recursive functions and effective Hausdorff dimension, Bull. Lond. Math. Soc. 43 (2011), no. 4, 636-654, DOI 10.1112/blms/bdr003. MR2820150 (2012g:03112)

[10] D. R. Hirschfeldt, C. G. Jockusch Jr., B. Kjos-Hanssen, S. Lempp, and T. A. Slaman, The strength of some combinatorial principles related to Ramsey's theorem for pairs, Computational Prospects of Infinity: Presented talks 14 (2008), 143-161.

[11] Jiayi Liu, $\mathrm{RT}_{2}^{2}$ does no imply $\mathrm{WKL}_{0}$, J. Symbolic Logic 77 (2012), no. 2, 609-620, DOI $10.2178 /$ jsl/1333566640. MR2963024

[12] Carl G. Jockusch Jr., Ramsey's theorem and recursion theory, J. Symbolic Logic 37 (1972), 268-280. MR0376319 (51 \#12495)

[13] Bjørn Kjos-Hanssen, Infinite subsets of random sets of integers, Math. Res. Lett. 16 (2009), no. 1, 103-110. MR2480564(2010b:03051)

[14] Bjørn Kjos-Hanssen, A strong law of computationally weak subsets, J. Math. Log. 11 (2011), no. 1, 1-10, DOI 10.1142/S0219061311000980. MR2833148

[15] Masahiro Kumabe and Andrew E. M. Lewis, A fixed-point-free minimal degree, J. Lond. Math. Soc. (2) 80 (2009), no. 3, 785-797, DOI 10.1112/jlms/jdp049. MR2559129 (2011d:03064) 
[16] Jack H. Lutz, Gales and the constructive dimension of individual sequences, Automata, languages and programming (Geneva, 2000), Lecture Notes in Comput. Sci., vol. 1853, Springer, Berlin, 2000, pp. 902-913, DOI 10.1007/3-540-45022-X_76. MR1795945 (2001g:68046)

[17] Elvira Mayordomo, A Kolmogorov complexity characterization of constructive Hausdorff dimension, Inform. Process. Lett. 84 (2002), no. 1, 1-3, DOI 10.1016/S0020-0190(02)00343-5. MR.1926330(2003h:68053)

[18] Joseph S. Miller, Extracting information is hard: a Turing degree of non-integral effective Hausdorff dimension, Adv. Math. 226 (2011), no. 1, 373-384, DOI 10.1016/j.aim.2010.06.024. MR2735764 (2012a:03114)

[19] André Nies, Computability and randomness, Oxford Logic Guides, vol. 51, Oxford University Press, Oxford, 2009. MR 2548883 (2011i:03003)

[20] F. P. Ramsey, On a Problem of Formal Logic, Proc. London Math. Soc. S2-30, no. 1, 264, DOI 10.1112/plms/s2-30.1.264. MR.1576401

[21] B. Ya. Ryabko, Coding of combinatorial sources and Hausdorff dimension (Russian), Dokl. Akad. Nauk SSSR 277 (1984), no. 5, 1066-1070. MR759967 (86g:94033)

[22] David Seetapun and Theodore A. Slaman, On the strength of Ramsey's theorem, Notre Dame J. Formal Logic 36 (1995), no. 4, 570-582, DOI 10.1305/ndjfl/1040136917. Special Issue: Models of arithmetic. MR 1368468 (96k:03136)

[23] Stephen G. Simpson, Subsystems of second order arithmetic, Perspectives in Mathematical Logic, Springer-Verlag, Berlin, 1999. MR1723993(2001i:03126)

Department of Mathematics, Central South University, ChangSha 410083, People's Republic OF ChinA

E-mail address: g.jiayi.liu@gmail.com 